Supporting Information for

\title{
Charge Transport and Quantum Interference Effects in Oxazole-Terminated Conjugated Oligomers
}

\author{
Songsong $\mathrm{Li}^{1,4 \dagger}$, Hao $\mathrm{Yu}^{2 \dagger}$, Kenneth Schwieter ${ }^{3}$, Kejia Chen ${ }^{2}$, Bo Li ${ }^{2}$, Yun Liu ${ }^{4}$, \\ Jeffrey S. Moore ${ }^{1,3,4}$, and Charles M. Schroeder ${ }^{1,2,3,4 *}$
}

${ }^{1}$ Department of Materials Science and Engineering, University of Illinois at Urbana-Champaign, Urbana, Illinois, 61801, United States

${ }^{2}$ Department of Chemical and Biomolecular Engineering, University of Illinois at UrbanaChampaign, Urbana, Illinois, 61801, United States

${ }^{3}$ Department of Chemistry, University of Illinois at Urbana-Champaign, Urbana, Illinois, 61801, United States

${ }^{4}$ Beckman Institute for Advanced Science and Technology, University of Illinois at UrbanaChampaign, Urbana, Illinois, 61801, United States

${ }^{\dagger}$ Contributed equally to this work.

*To whom correspondence should be addressed. Email: cms@illinois.edu

Table of Contents:

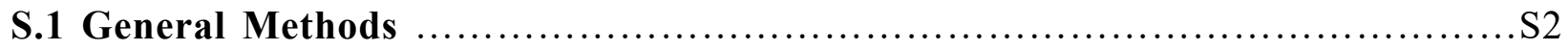

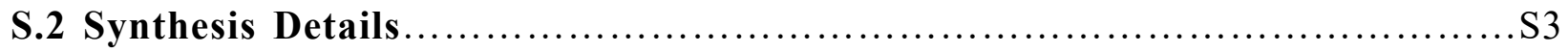

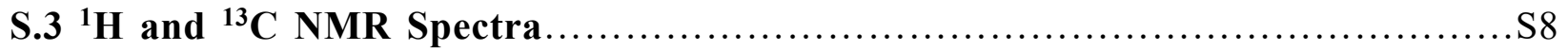

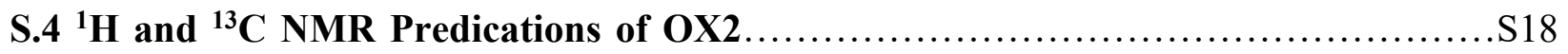

S.5 Additional Experimental Data and Theoretical Calculations....................S19 


\section{S.1 General Methods}

Materials. All reagents were purchased from commercial suppliers and used as received unless otherwise stated. Dry dimethylformamide (DMF), dichloromethane (DCM) and tetrahydrofuran (THF) were obtained from a Solvent Delivery System (SDS) equipped with activated neutral alumina columns under argon. Column chromatography were performed on Biotage Isolera System using Silicycle Siliasep HP flash cartridges.

\section{Characterization}

NMR. ${ }^{1} \mathrm{H}$ and ${ }^{13} \mathrm{C}$ NMR spectra $(500 \mathrm{MHz}$ and $125 \mathrm{MHz}$ ) were recorded at room temperature (298 $\mathrm{K})$. Chemical shifts are reported in $\delta(\mathrm{ppm})$ referenced on residual solvent peaks. Coupling constants $(\mathrm{J})$ are expressed in Hertz $(\mathrm{Hz})$. Splitting patterns are designated as: s (singlet), $\mathrm{d}$ (doublet), $\mathrm{t}$ (triplet), $\mathrm{m}$ (multiplet).

Mass-Spectrometry. High resolution EI and ESI mass spectra were recorded on a Micromass 70VSE spectrometer and Micromass Q-TOF Ultima spectrometer. 


\section{S.2 Synthesis Details}

Compound $\mathrm{OX} 3, \mathbf{O X} 4, \mathbf{O}_{4}-\mathbf{m}-\mathbf{O}_{4}{ }^{\mathrm{S} 1}, \mathbf{F U 1}{ }^{\mathrm{S} 2}$ were synthesized according to literature procedures. Characterization data matched literature.

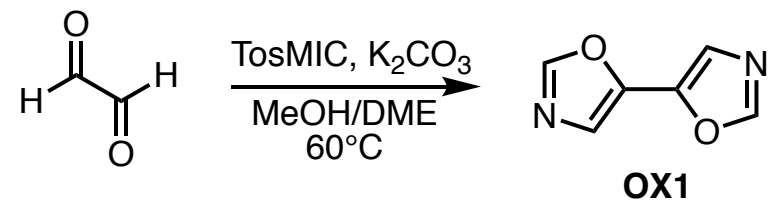

5,5'-Bioxazole (OX1). To a glass vial was added glyoxal ( $40 \%$ wt in $\left.\mathrm{H}_{2} \mathrm{O}\right)(73 \mu \mathrm{L}, 0.50 \mathrm{mmol})$, TosMIC (195 mg, $1.00 \mathrm{mmol}), \mathrm{K}_{2} \mathrm{CO}_{3}(414 \mathrm{mg}, 3.00 \mathrm{mmol}), \mathrm{MeOH}(3 \mathrm{~mL})$, and DME (1.5 mL). The reaction mixture was stirred at $60^{\circ} \mathrm{C}$ for $16 \mathrm{~h}$. After cooling to room temperature, the mixture was diluted with $\mathrm{H}_{2} \mathrm{O}(50 \mathrm{~mL})$ and extracted three times with $\mathrm{CH}_{2} \mathrm{Cl}_{2}(50 \mathrm{~mL} \times 3)$. The combined organic extracts were dried $\left(\mathrm{MgSO}_{4}\right)$ and concentrated. Flash column chromatography $\left(\mathrm{SiO}_{2}, 40-\right.$ $60 \%$ ethyl acetate in hexanes) yielded the product as a white solid (20 mg, 29\%). ${ }^{1} \mathrm{H}$ NMR (500 $\left.\mathrm{MHz}, \mathrm{CDCl}_{3}\right) \delta 7.96(\mathrm{~s}, 2 \mathrm{H}), 7.39(\mathrm{~s}, 2 \mathrm{H}) ;{ }^{13} \mathrm{C} \mathrm{NMR}\left(125 \mathrm{MHz}, \mathrm{CDCl}_{3}\right) \delta 150.86,141.03,123.77$. HRMS (EI): Exact mass calcd for $\mathrm{C}_{6} \mathrm{H}_{5} \mathrm{~N}_{2} \mathrm{O}_{2}[\mathrm{M}+\mathrm{H}]^{+}$137.0351, found 137.0351.

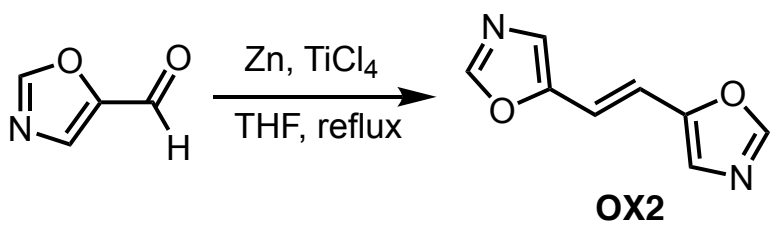

(E)-1,2-bis(oxazol-5-yl)ethene (OX2). To a flame-dried flask was added Znic powder (273 $\mathrm{mg}$, $4.2 \mathrm{mmol})$ and dry THF $(10 \mathrm{~mL})$. At $0{ }^{\circ} \mathrm{C}, \mathrm{TiCl}_{4}(0.22 \mathrm{~mL}, 2 \mathrm{mmol})$ was added dropwise to the suspension. The reaction mixture was stirred at $60^{\circ} \mathrm{C}$ for $0.5 \mathrm{~h}$. Oxazole-5-carbaldehyde $(100 \mathrm{mg}$, $1 \mathrm{mmol})$ was then dissolved in dry THF $(5 \mathrm{~mL})$ and added to resulting mixture slowly. The resulting mixture was stirred at $60{ }^{\circ} \mathrm{C}$ for $16 \mathrm{~h}$ before quenching with sat. $\mathrm{K}_{2} \mathrm{CO}_{3}$ aqueous solution $(50 \mathrm{~mL})$. Undissolved solids were removed by filtering, the filtrate was extracted three times with ethyl acetate $(50 \mathrm{~mL} \times 3)$. The combined organic extracts were dried $\left(\mathrm{MgSO}_{4}\right)$ and concentrated. Flash column chromatography ( $\mathrm{SiO}_{2}, 30-60 \%$ ethyl acetate in hexanes) yielded the product as a white solid (30 mg, 37\%). ${ }^{1} \mathrm{H}$ NMR (500 MHz, $\left.\mathrm{CDCl}_{3}\right) \delta 7.88(\mathrm{~s}, 2 \mathrm{H}), 7.14(\mathrm{~s}, 2 \mathrm{H}), 6.93(\mathrm{~s}, 2 \mathrm{H})$; ${ }^{13} \mathrm{C}$ NMR $\left(125 \mathrm{MHz}, \mathrm{CDCl}_{3}\right) \delta 151.02,125.83,124.91,114.29$; HRMS (ESI): Exact mass calcd for $\mathrm{C}_{8} \mathrm{H}_{7} \mathrm{~N}_{2} \mathrm{O}_{2}[\mathrm{M}+\mathrm{H}]^{+}$163.0508, found 163.0501.

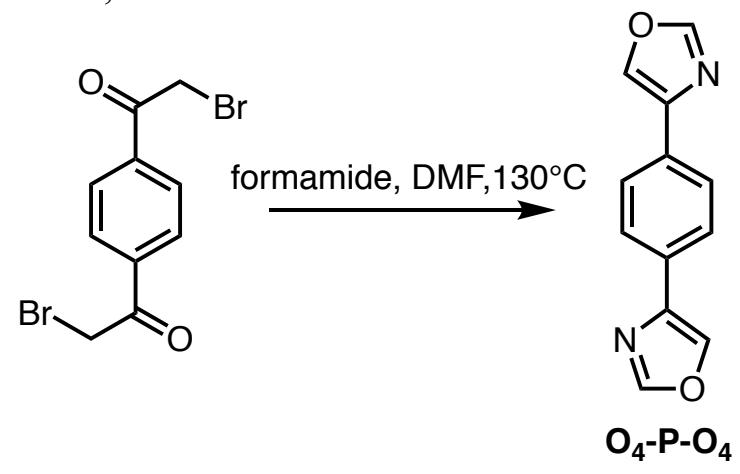

1,4-bis(oxazol-4-yl)benzene ( $\left.\mathbf{O}_{4}-\mathbf{p}-\mathbf{O}_{4}\right) .1$ 1, 1'-(1,4-phenylene)bis(2-bromoethan-1-one) (100 mg, $0.315 \mathrm{mmol})$ was dissolved in dry DMF $(2 \mathrm{~mL})$ and formamide $(2 \mathrm{~mL})$ and heated at $130^{\circ} \mathrm{C}$ for 4 h. After cooling to room temperature, the mixture was diluted with $\mathrm{H}_{2} \mathrm{O}(50 \mathrm{~mL})$ and extracted 
three times with $\mathrm{CH}_{2} \mathrm{Cl}_{2}(50 \mathrm{~mL} \times 3)$. The combined organic extracts were dried $\left(\mathrm{MgSO}_{4}\right)$ and concentrated. Flash column chromatography $\left(\mathrm{SiO}_{2}, 10-40 \%\right.$ ethyl acetate in hexanes) yielded the product as a white solid (20 mg, 30\%). ${ }^{1} \mathrm{H}$ NMR $\left(500 \mathrm{MHz}, \mathrm{CDCl}_{3}\right) \delta 8.01(\mathrm{~s}, 1 \mathrm{H}), 7.98(\mathrm{~s}, 1 \mathrm{H})$, $7.84(\mathrm{~s}, 2 \mathrm{H}) .{ }^{13} \mathrm{C} \mathrm{NMR}\left(125 \mathrm{MHz}, \mathrm{CDCl}_{3}\right) \delta 151.61,140.29,134.14,130.76,126.22$. HRMS (ESI): Exact mass calcd for $\mathrm{C}_{12} \mathrm{H}_{9} \mathrm{~N}_{2} \mathrm{O}_{2}[\mathrm{M}+\mathrm{H}]^{+} 213.0664$, found 213.0664 .

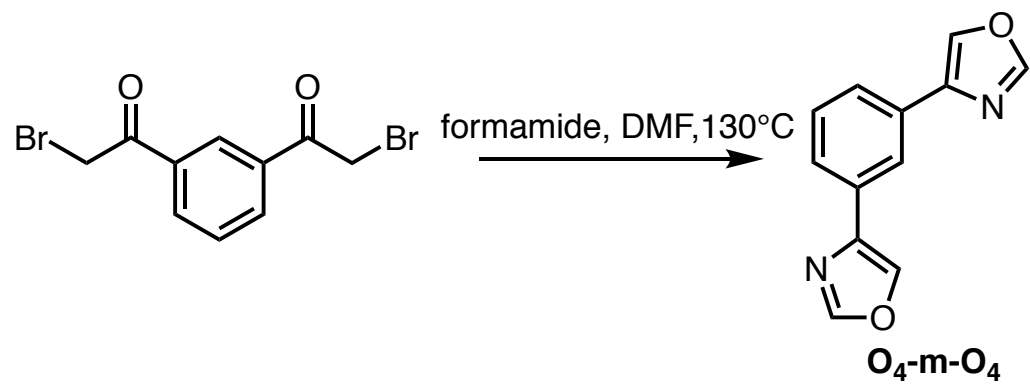

1,3-bis(oxazol-4-yl)benzene ( $\mathbf{O}_{4}$-m-O $\left.\mathbf{O}_{4}\right)$. 1,1'-(1,3-phenylene)bis(2-bromoethan-1-one) (200 mg, $0.315 \mathrm{mmol})$ was dissolved in dry DMF $(2 \mathrm{~mL})$ and formamide $(2 \mathrm{~mL})$ and heated at $130{ }^{\circ} \mathrm{C}$ for 4 h. After cooling to room temperature, the mixture was diluted with $\mathrm{H}_{2} \mathrm{O}(50 \mathrm{~mL})$ and extracted three times with $\mathrm{CH}_{2} \mathrm{Cl}_{2}(50 \mathrm{~mL} \times 3)$. The combined organic extracts were dried $\left(\mathrm{MgSO}_{4}\right)$ and concentrated. Flash column chromatography $\left(\mathrm{SiO}_{2}, 10-40 \%\right.$ ethyl acetate in hexanes) yielded the product as a white solid (30 mg, 44\%). ${ }^{1} \mathrm{H}$ NMR $\left(500 \mathrm{MHz}, \mathrm{CDCl}_{3}\right) \delta 8.16(\mathrm{~s}, 1 \mathrm{H}), 8.05(\mathrm{~s}, 2 \mathrm{H})$, $7.99(\mathrm{~s}, 2 \mathrm{H}), 7.76(\mathrm{~d}, J=7.7 \mathrm{~Hz}, 2 \mathrm{H}), 7.51$ (t, $J=7.7 \mathrm{~Hz}, 1 \mathrm{H}) \cdot{ }^{13} \mathrm{C}$ NMR $\left(125 \mathrm{MHz}, \mathrm{CDCl}_{3}\right) \delta$ 151.32, 140.12, 134.08, 131.32, 129.34, 125.41, 122.72. HRMS (ESI): Exact mass calcd for $\mathrm{C}_{12} \mathrm{H}_{9} \mathrm{~N}_{2} \mathrm{O}_{2}[\mathrm{M}+\mathrm{H}]^{+}$213.0664, found 213.0666.

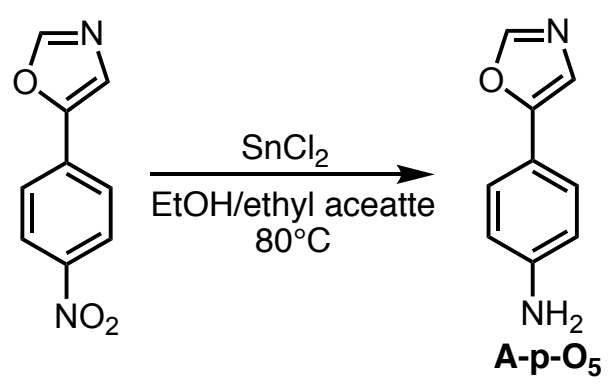

4-(oxazol-5-yl)aniline (A-p-O5). To a flask was added 5-(4-nitrophenyl)oxazole (300 mg, 1.58 $\mathrm{mmol}), \mathrm{SnCl}_{2}(1244 \mathrm{mg}, 6.56 \mathrm{mmol}), \mathrm{EtOH}(10 \mathrm{~mL})$, and ethyl acetate $(20 \mathrm{~mL})$. The reaction mixture was stirred at $80{ }^{\circ} \mathrm{C}$ for $16 \mathrm{~h}$. After cooling to room temperature, the mixture was neutralized with sat. $\mathrm{NaHCO}_{3}$ aqueous solution $(100 \mathrm{~mL})$ and extracted three times with ethyl acetate $(50 \mathrm{~mL} \times 3)$. The combined organic extracts were dried $\left(\mathrm{MgSO}_{4}\right)$ and concentrated. Flash column chromatography $\left(\mathrm{SiO}_{2}, 40 \%\right.$ ethyl acetate in hexanes) yielded the product as a light yellow solid (200 mg, 80\%). Characterization data matched literature. ${ }^{\mathrm{S} 3}$ 


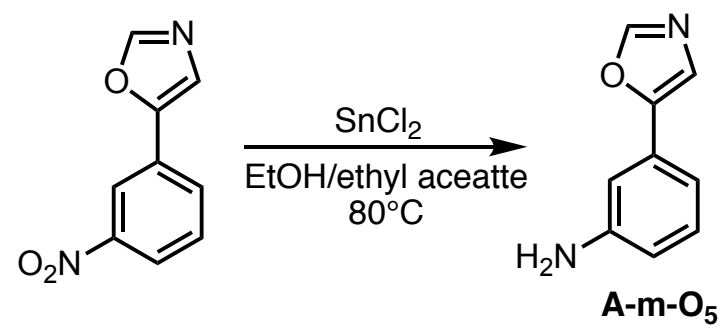

3-(oxazol-5-yl)aniline (A-m-O $\left.\mathbf{O}_{5}\right)$. To a flask was added 5-(3-nitrophenyl)oxazole (300 mg, 1.58 $\mathrm{mmol}), \mathrm{SnCl}_{2}(1244 \mathrm{mg}, 6.56 \mathrm{mmol}), \mathrm{EtOH}(10 \mathrm{~mL})$, and ethyl acetate $(20 \mathrm{~mL})$. The reaction mixture was stirred at $80{ }^{\circ} \mathrm{C}$ for $16 \mathrm{~h}$. After cooling to room temperature, the mixture was neutralized with sat. $\mathrm{NaHCO}_{3}$ aqueous solution $(100 \mathrm{~mL})$ and extracted three times with ethyl acetate $(50 \mathrm{~mL} \times 3)$. The combined organic extracts were dried $\left(\mathrm{MgSO}_{4}\right)$ and concentrated. Flash column chromatography $\left(\mathrm{SiO}_{2}, 40 \%\right.$ ethyl acetate in hexanes) yielded the product as a light yellow solid (200 mg, 80\%). Characterization data matched literature. ${ }^{\mathrm{S4}}$

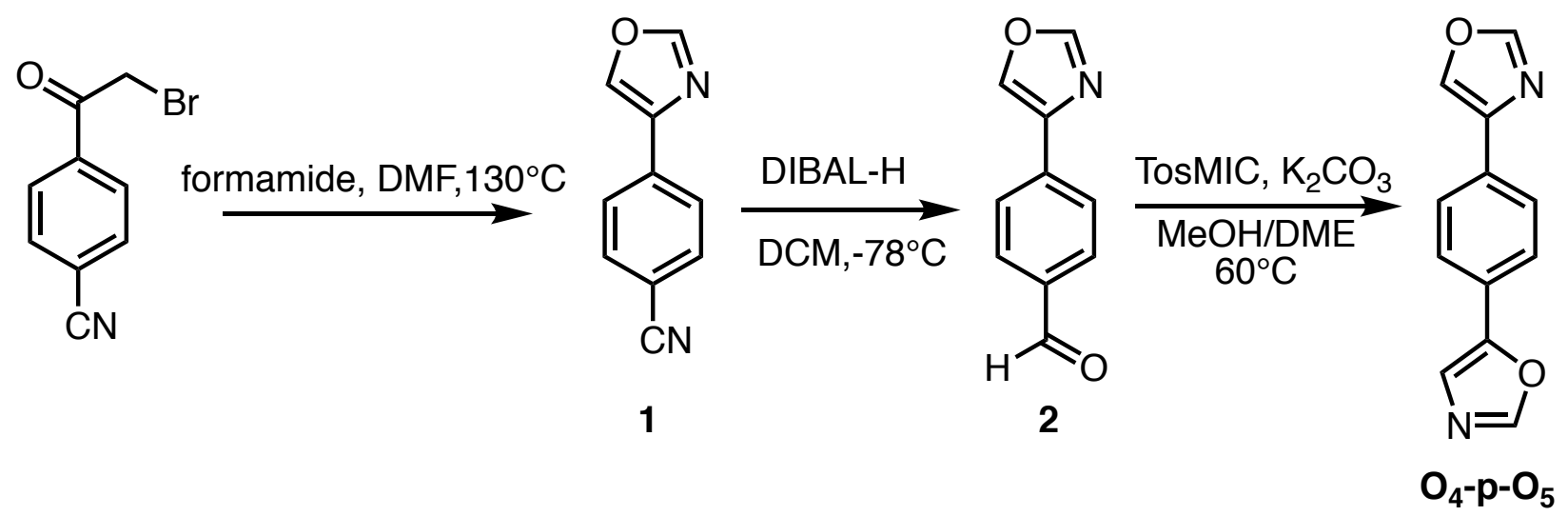

4-(oxazol-4-yl)benzonitrile (1). 4-(2-bromoacetyl)benzonitrile ( $1 \mathrm{~g}, 4.7 \mathrm{mmol})$ was dissolved in dry DMF $(5 \mathrm{~mL})$ and formamide $(5 \mathrm{~mL})$ and heated at $130{ }^{\circ} \mathrm{C}$ for $4 \mathrm{~h}$. After cooling to room temperature, the mixture was diluted with $\mathrm{H}_{2} \mathrm{O}(100 \mathrm{~mL})$ and extracted three times with $\mathrm{CH}_{2} \mathrm{Cl}_{2}$ $(50 \mathrm{~mL} \times 3)$. The combined organic extracts were dried $\left(\mathrm{MgSO}_{4}\right)$ and concentrated. Flash column chromatography $\left(\mathrm{SiO}_{2}, 10-30 \%\right.$ ethyl acetate in hexanes) yielded the product as a light yellow solid (200 mg, 26\%). ${ }^{1} \mathrm{H}$ NMR (500 MHz, $\left.\mathrm{CDCl}_{3}\right) \delta 8.08(\mathrm{~s}, 1 \mathrm{H}), 8.00(\mathrm{~s}, 1 \mathrm{H}), 7.88(\mathrm{~d}, \mathrm{~J}=7.8$ $\mathrm{Hz}, 2 \mathrm{H}), 7.72(\mathrm{~d}, \mathrm{~J}=7.5 \mathrm{~Hz}, 2 \mathrm{H}) .{ }^{13} \mathrm{C} \mathrm{NMR}\left(125 \mathrm{MHz}, \mathrm{CDCl}_{3}\right) \delta 151.32,140.12,134.08,131.32$, 129.34, 125.41, 122.72. HRMS (ESI): Exact mass calcd for $\mathrm{C}_{10} \mathrm{H}_{7} \mathrm{~N}_{2} \mathrm{O}[\mathrm{M}+\mathrm{H}]^{+}$171.0558, found 171.0564 .

4-(oxazol-4-yl)benzaldehyde (2). 4-(oxazol-4-yl)benzonitrile ( $80 \mathrm{mg}, 0.47 \mathrm{mmol})$ was dissolved in dry DCM $(5 \mathrm{~mL})$ and cooled down to $-78^{\circ} \mathrm{C} .1 \mathrm{M}$ DIBAL-H solution in heptane $(0.5,0.5 \mathrm{mmol})$ was slowly added to mixture. After $1 \mathrm{~h}$, the resulting mixture was quenched with $1 \mathrm{M} \mathrm{HCl}$ and extracted three times with $\mathrm{CH}_{2} \mathrm{Cl}_{2}(25 \mathrm{~mL} \times 3)$. The combined organic extracts were dried $\left(\mathrm{MgSO}_{4}\right)$ and concentrated. Flash column chromatography $\left(\mathrm{SiO}_{2}, 10-30 \%\right.$ ethyl acetate in hexanes) yielded the product as a light yellow solid $(70 \mathrm{mg}, 87 \%) .{ }^{1} \mathrm{H}$ NMR $\left(500 \mathrm{MHz}, \mathrm{CDCl}_{3}\right) \delta 10.03(\mathrm{~s}, 1 \mathrm{H})$, 8.08 (s, 1H), 7.99 (s, 1H), 7.93 (s, 4H). ${ }^{13} \mathrm{C}$ NMR $\left(125 \mathrm{MHz}, \mathrm{CDCl}_{3}\right) \delta 191.63,151.66,139.48$, 136.53, 135.91, 135.37, 130.33, 126.00. HRMS (ESI): Exact mass calcd for $\mathrm{C}_{10} \mathrm{H}_{8} \mathrm{NO}_{2}[\mathrm{M}+\mathrm{H}]^{+}$ 174.0555, found 174.0555 . 
5-(4-(oxazol-4-yl)phenyl)oxazole ( $\left.\mathbf{O}_{4}-\mathbf{p}-\mathbf{O}_{5}\right)$. To a glass vial was added 4-(oxazol-4yl)benzaldehyde (70 mg, $0.41 \mathrm{mmol})$, TosMIC (90 mg, $0.46 \mathrm{mmol}), \mathrm{K}_{2} \mathrm{CO}_{3}(110 \mathrm{mg}, 0.80 \mathrm{mmol})$, $\mathrm{MeOH}(10 \mathrm{~mL})$, and DME $(3 \mathrm{~mL})$. The reaction mixture was stirred at $60^{\circ} \mathrm{C}$ for $16 \mathrm{~h}$. After cooling to room temperature, the mixture was diluted with $\mathrm{H}_{2} \mathrm{O}(50 \mathrm{~mL})$ and extracted three times with $\mathrm{CH}_{2} \mathrm{Cl}_{2}(50 \mathrm{~mL} \times 3)$. The combined organic extracts were dried $\left(\mathrm{MgSO}_{4}\right)$ and concentrated. Flash column chromatography $\left(\mathrm{SiO}_{2}, 20-40 \%\right.$ ethyl acetate in hexanes) yielded the product as a white solid (70 mg, 80\%). ${ }^{1} \mathrm{H}$ NMR (500 MHz, $\left.\mathrm{CDCl}_{3}\right) \delta 8.02(\mathrm{~s}, 1 \mathrm{H}), 7.97(\mathrm{~d}, \mathrm{~J}=14.6 \mathrm{~Hz}, 2 \mathrm{H}), 7.85$ $(\mathrm{d}, \mathrm{J}=8.5 \mathrm{~Hz}, 2 \mathrm{H}), 7.74(\mathrm{~d}, \mathrm{~J}=8.5 \mathrm{~Hz}, 2 \mathrm{H}), 7.42(\mathrm{~s}, 1 \mathrm{H}) .{ }^{13} \mathrm{C} \mathrm{NMR}\left(125 \mathrm{MHz}, \mathrm{CDCl}_{3}\right) \delta 151.44$, 151.23, 150.52, 139.83, 134.11, 130.96, 127.43, 126.09, 124.79, 121.76. HRMS (EI): Exact mass calcd for $\mathrm{C}_{12} \mathrm{H}_{9} \mathrm{~N}_{2} \mathrm{O}_{2}[\mathrm{M}+\mathrm{H}]^{+}$213.0668, found 213.0668.

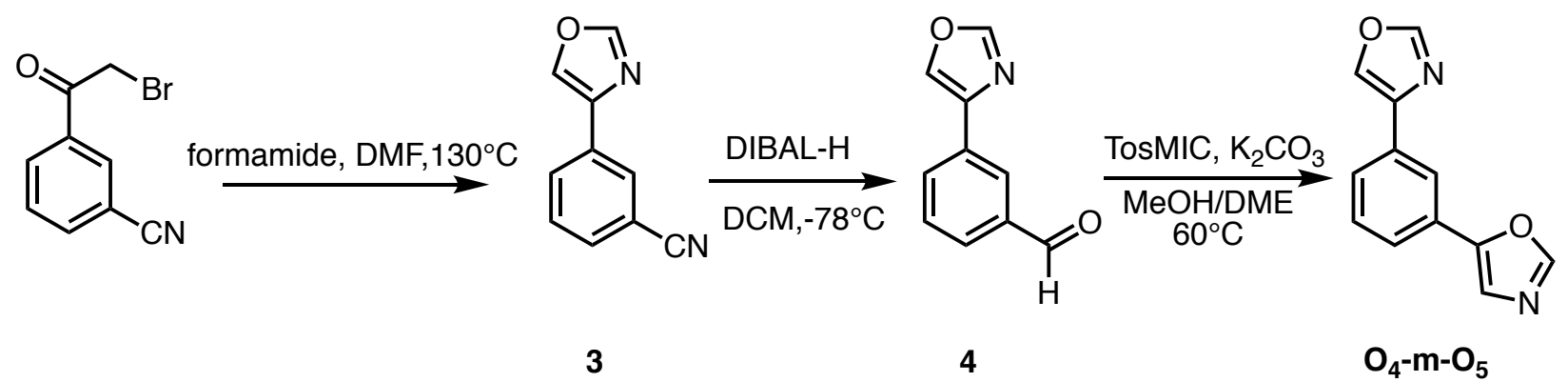

3-(oxazol-4-yl)benzonitrile (3). 3-(2-bromoacetyl)benzonitrile (1.5 g, $6.7 \mathrm{mmol})$ was dissolved in dry DMF $(5 \mathrm{~mL})$ and formamide $(5 \mathrm{~mL})$ and heated at $130{ }^{\circ} \mathrm{C}$ for $4 \mathrm{~h}$. After cooling to room temperature, the mixture was diluted with $\mathrm{H}_{2} \mathrm{O}(100 \mathrm{~mL})$ and extracted three times with $\mathrm{CH}_{2} \mathrm{Cl}_{2}$ $(50 \mathrm{~mL} \times 3)$. The combined organic extracts were dried $\left(\mathrm{MgSO}_{4}\right)$ and concentrated. Flash column chromatography $\left(\mathrm{SiO}_{2}, 10-30 \%\right.$ ethyl acetate in hexanes) yielded the product as a white solid (400 $\mathrm{mg}, 35 \%) .{ }^{1} \mathrm{H}$ NMR $\left(500 \mathrm{MHz}, \mathrm{CDCl}_{3}\right) \delta 8.08(\mathrm{~s}, 1 \mathrm{H}), 8.05(\mathrm{~s}, 1 \mathrm{H}), 8.02-7.98(\mathrm{~m}, 2 \mathrm{H}), 7.63(\mathrm{~d}$, $\mathrm{J}=7.7 \mathrm{~Hz}, 1 \mathrm{H}), 7.55(\mathrm{t}, \mathrm{J}=7.8 \mathrm{~Hz}, 1 \mathrm{H}) .{ }^{13} \mathrm{C} \mathrm{NMR}\left(125 \mathrm{MHz}, \mathrm{CDCl}_{3}\right) \delta 151.69,138.67,134.65$, 132.11, 131.53, 129.69, 129.66, 129.15, 118.50, 113.15. HRMS (ESI): Exact mass calcd for $\mathrm{C}_{10} \mathrm{H}_{7} \mathrm{~N}_{2} \mathrm{O}[\mathrm{M}+\mathrm{H}]^{+}$171.0558, found 171.0563.

3-(oxazol-4-yl)benzaldehyde (4). 3-(oxazol-4-yl)benzonitrile (100 mg, $0.58 \mathrm{mmol}$ ) was dissolved in dry DCM $(5 \mathrm{~mL})$ and cooled down to $-78^{\circ} \mathrm{C} .1 \mathrm{M}$ DIBAL-H solution in heptane $(0.6,0.6 \mathrm{mmol})$ was slowly added to mixture. After $1 \mathrm{~h}$, the resulting mixture was quenched with $1 \mathrm{M} \mathrm{HCl}$ and extracted three times with $\mathrm{CH}_{2} \mathrm{Cl}_{2}(25 \mathrm{~mL} \times 3)$. The combined organic extracts were dried $\left(\mathrm{MgSO}_{4}\right)$ and concentrated. Flash column chromatography $\left(\mathrm{SiO}_{2}, 10-30 \%\right.$ ethyl acetate in hexanes) yielded the product as a light yellow solid $(50 \mathrm{mg}, 50 \%) .{ }^{1} \mathrm{H} \mathrm{NMR}\left(500 \mathrm{MHz}, \mathrm{CDCl}_{3}\right) \delta 10.09(\mathrm{~s}, 1 \mathrm{H})$, $8.27(\mathrm{~s}, 1 \mathrm{H}), 8.06(\mathrm{~d}, \mathrm{~J}=13.4 \mathrm{~Hz}, 2 \mathrm{H}), 8.00(\mathrm{~s}, 1 \mathrm{H}), 7.87(\mathrm{~d}, \mathrm{~J}=7.6 \mathrm{~Hz}, 1 \mathrm{H}), 7.62(\mathrm{t}, \mathrm{J}=7.7 \mathrm{~Hz}$, 1H). ${ }^{13} \mathrm{C}$ NMR $\left(125 \mathrm{MHz}, \mathrm{CDCl}_{3}\right) \delta 192.04,151.56,139.35,136.88,134.46,131.83,131.34$, 129.57, 129.21, 126.77. HRMS (ESI): Exact mass calcd for $\mathrm{C}_{10} \mathrm{H}_{8} \mathrm{NO}_{2}[\mathrm{M}+\mathrm{H}]^{+} 174.0555$, found 174.0558 .

5-(3-(oxazol-4-yl)phenyl)oxazole $\quad\left(\mathbf{O}_{4}-\mathbf{m}-\mathbf{O}_{5}\right)$. To a glass vial was added 3-(oxazol-4yl)benzaldehyde (30 mg, $0.17 \mathrm{mmol}$ ), TosMIC (40 mg, $0.21 \mathrm{mmol}), \mathrm{K}_{2} \mathrm{CO}_{3}(48 \mathrm{mg}, 0.35 \mathrm{mmol})$, $\mathrm{MeOH}(5 \mathrm{~mL})$, and DME $(1.5 \mathrm{~mL})$. The reaction mixture was stirred at $60{ }^{\circ} \mathrm{C}$ for $16 \mathrm{~h}$. After cooling to room temperature, the mixture was diluted with $\mathrm{H}_{2} \mathrm{O}(50 \mathrm{~mL})$ and extracted three times with $\mathrm{CH}_{2} \mathrm{Cl}_{2}(50 \mathrm{~mL} \times 3)$. The combined organic extracts were dried $\left(\mathrm{MgSO}_{4}\right)$ and concentrated. Flash column chromatography $\left(\mathrm{SiO}_{2}, 20-40 \%\right.$ ethyl acetate in hexanes) yielded the product as a 
white solid (30 mg, 81\%). ${ }^{1} \mathrm{H}$ NMR $\left(500 \mathrm{MHz}, \mathrm{CDCl}_{3}\right) \delta 8.10(\mathrm{~s}, 1 \mathrm{H}), 8.05(\mathrm{~d}, \mathrm{~J}=2.6 \mathrm{~Hz}, 1 \mathrm{H})$, $8.00(\mathrm{~d}, \mathrm{~J}=2.6 \mathrm{~Hz}, 1 \mathrm{H}), 7.97(\mathrm{~d}, \mathrm{~J}=2.8 \mathrm{~Hz}, 1 \mathrm{H}), 7.73(\mathrm{~d}, \mathrm{~J}=7.9 \mathrm{~Hz}, 1 \mathrm{H}), 7.65(\mathrm{~d}, \mathrm{~J}=7.7 \mathrm{~Hz}$, $1 \mathrm{H}), 7.54-7.48(\mathrm{~m}, 1 \mathrm{H}), 7.46(\mathrm{~d}, \mathrm{~J}=2.7 \mathrm{~Hz}, 1 \mathrm{H}) .{ }^{13} \mathrm{C}$ NMR $\left(125 \mathrm{MHz}, \mathrm{CDCl}_{3}\right) \delta 151.67,151.45$, $150.81,140.07,134.41,131.76,129.67,128.60,125.95,124.33,122.19,121.76$. HRMS (EI): Exact mass calcd for $\mathrm{C}_{12} \mathrm{H}_{9} \mathrm{~N}_{2} \mathrm{O}_{2}[\mathrm{M}+\mathrm{H}]^{+}$213.0664, found 213.0668. 


\section{S.3 ${ }^{1} \mathrm{H}$ and ${ }^{13} \mathrm{C}$ NMR Spectra}<smiles>c1ncc(-c2cnco2)o1</smiles>

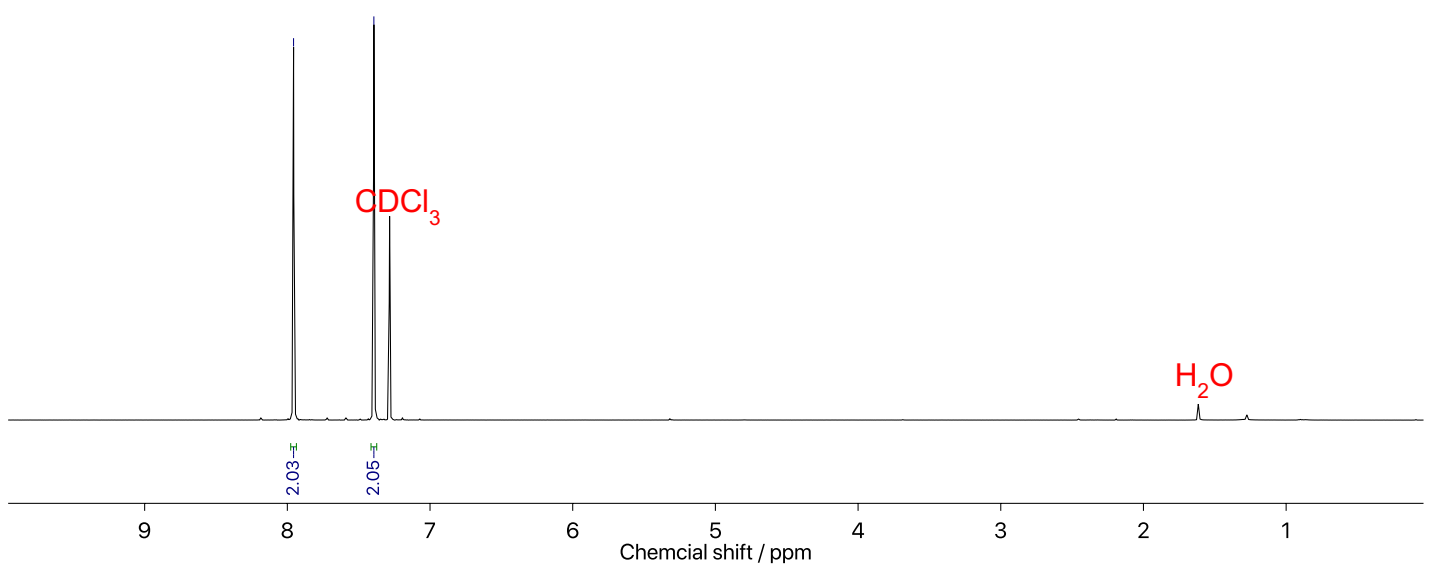

Figure S1. ${ }^{1} \mathrm{H}$ NMR spectrum of $\mathbf{O X 1}\left(500 \mathrm{MHz}, \mathrm{CDCl}_{3}\right)$.

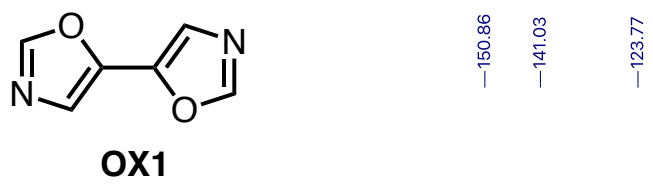



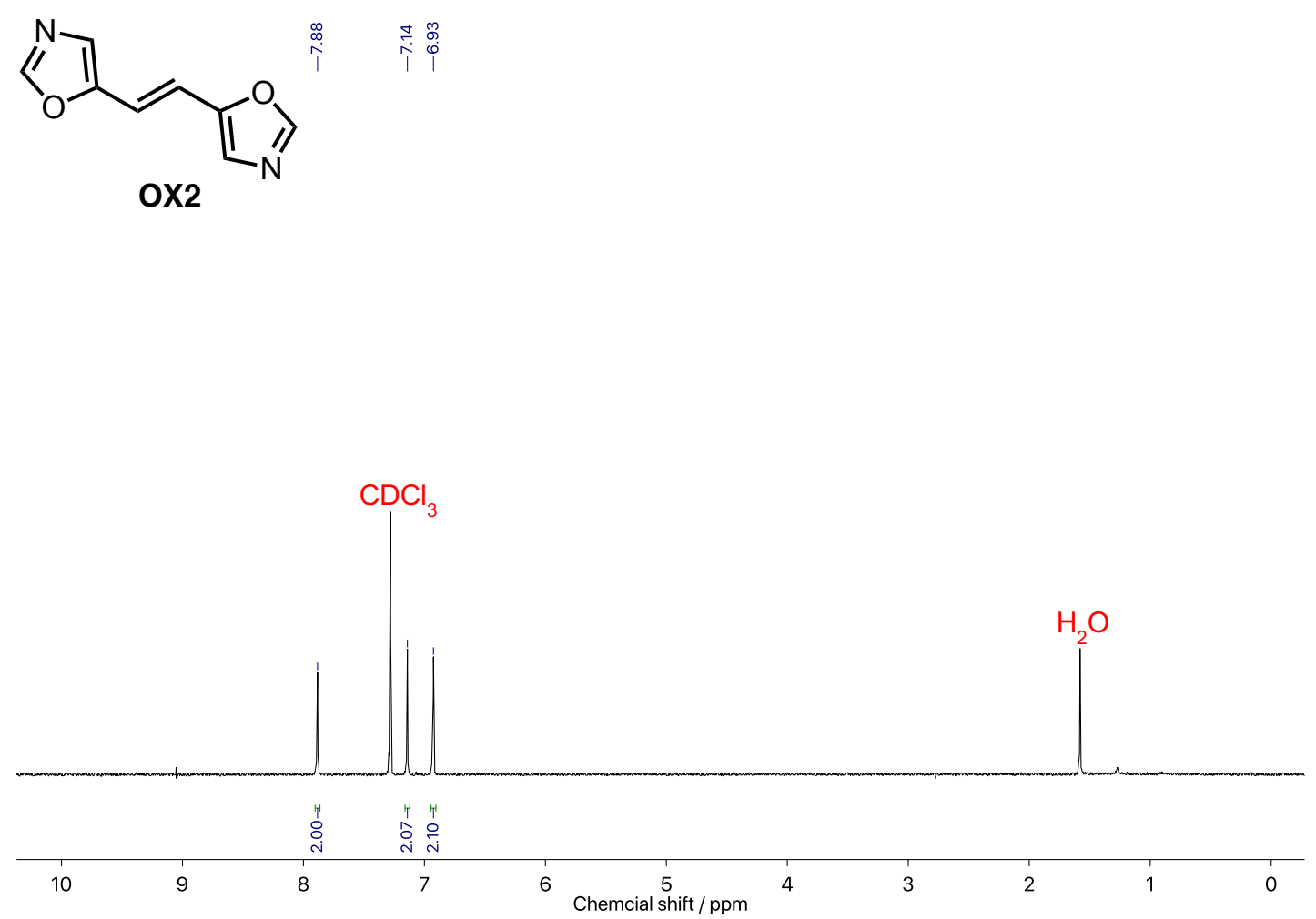

Figure S3. ${ }^{1} \mathrm{H}$ NMR spectrum of $\mathbf{O X 2}\left(500 \mathrm{MHz}^{\mathrm{CDCl}}{ }_{3}\right)$.<smiles>[O]O[Na]</smiles>

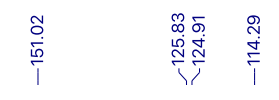

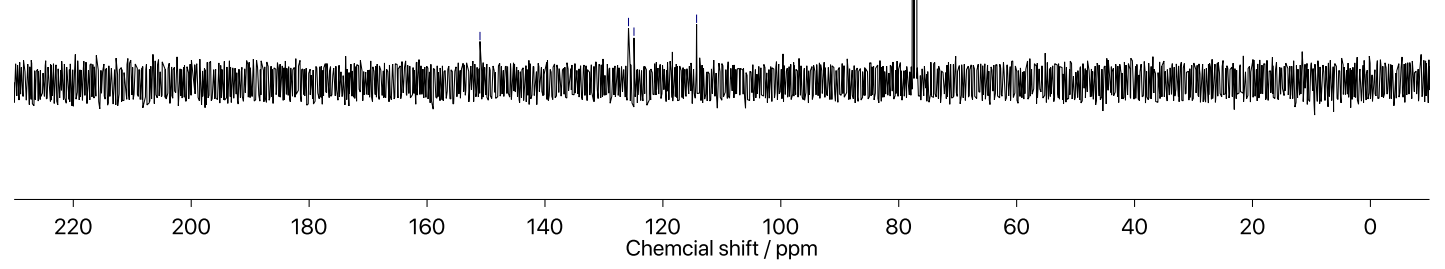

Figure S4. ${ }^{13} \mathrm{C}$ NMR spectrum of $\mathbf{O X 2}\left(125 \mathrm{MHz}, \mathrm{CDCl}_{3}\right)$. 

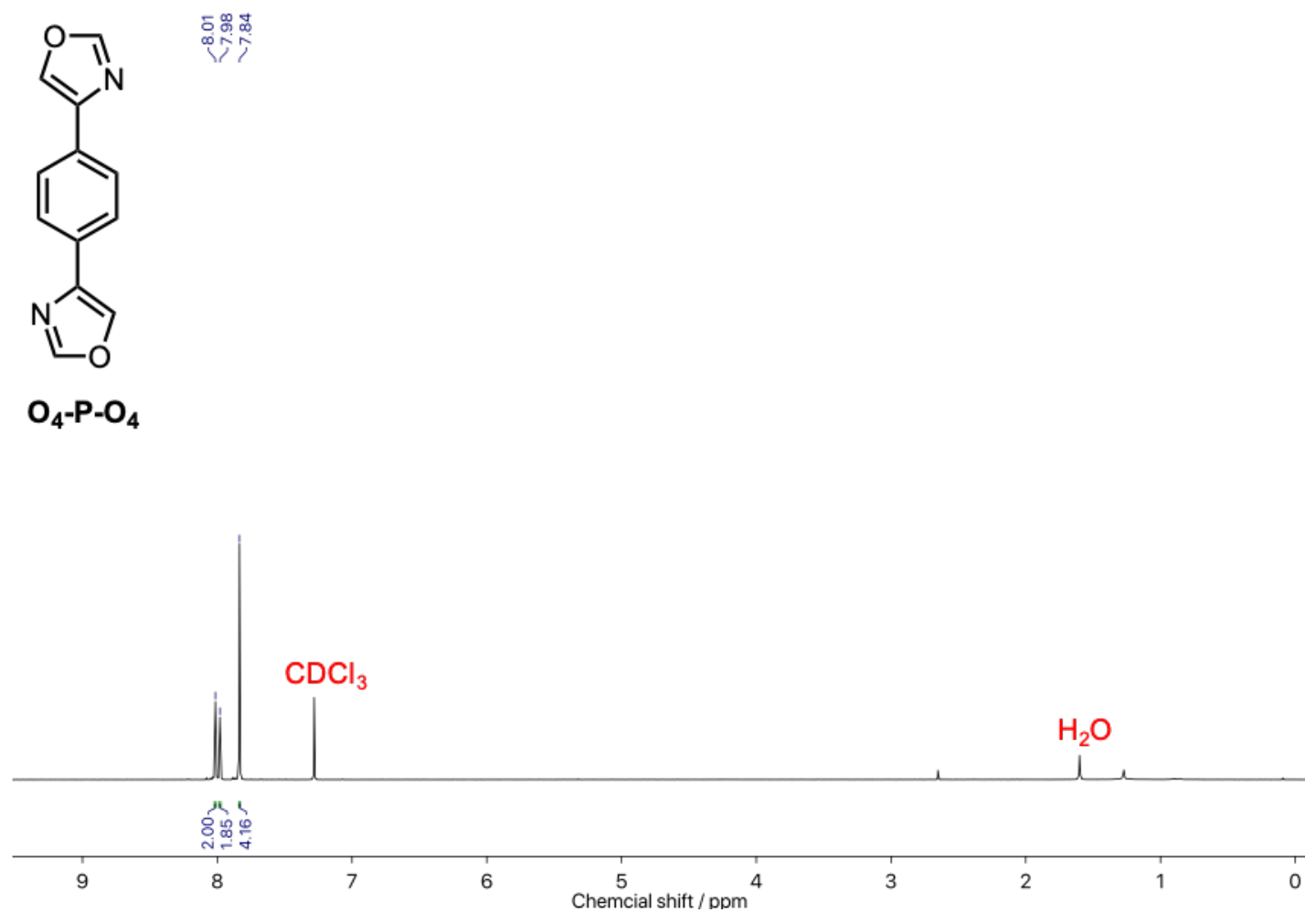

Figure S5. ${ }^{1} \mathrm{H}$ NMR spectrum of $\mathbf{O}_{4}-\mathbf{p}-\mathbf{O}_{4}\left(500 \mathrm{MHz}, \mathrm{CDCl}_{3}\right)$.
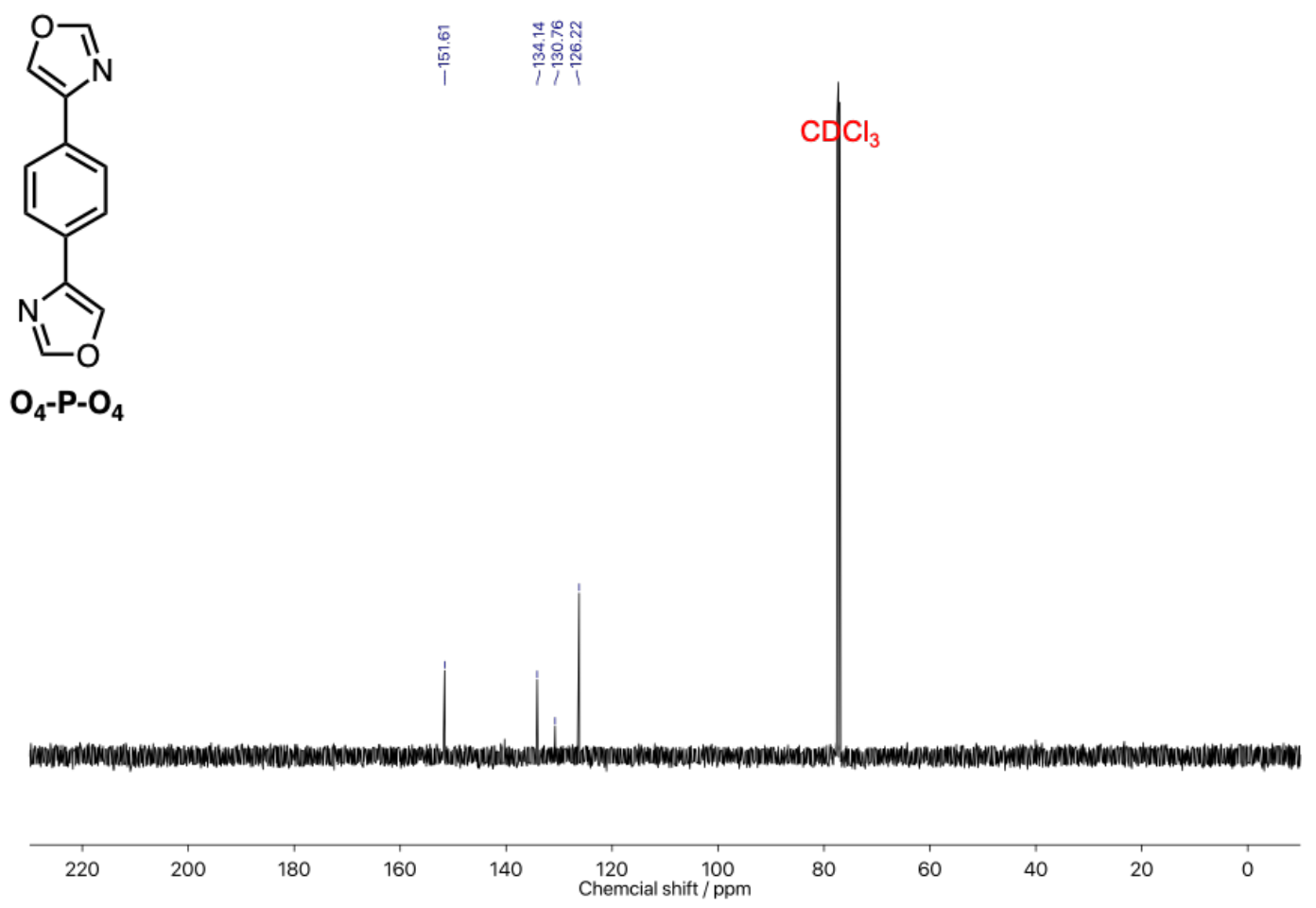

Figure S6. ${ }^{13} \mathrm{C}$ NMR spectrum of $\mathbf{O}_{4}-\mathbf{p}-\mathbf{O}_{4}\left(125 \mathrm{MHz}, \mathrm{CDCl}_{3}\right)$. 


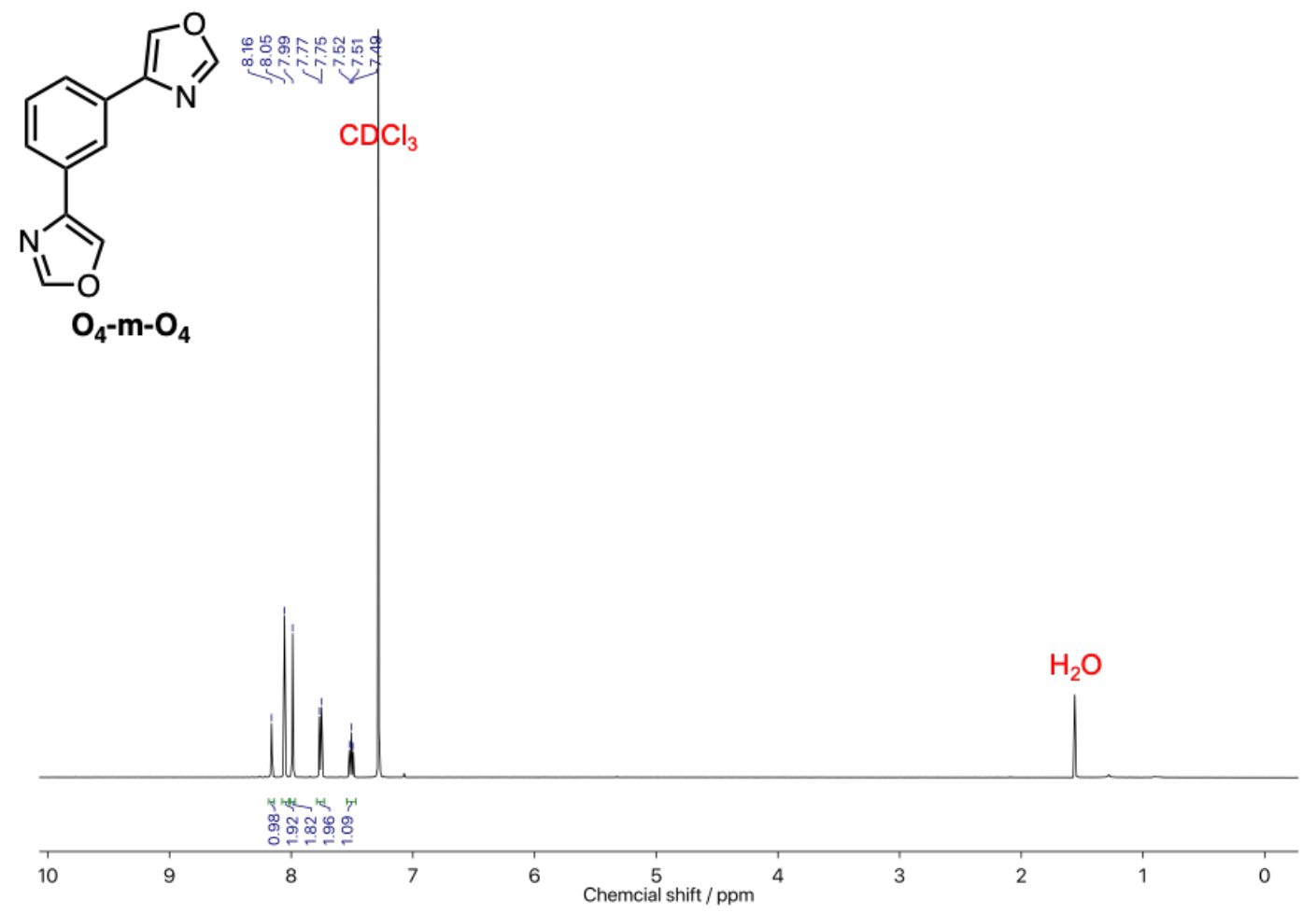

Figure S7. ${ }^{1} \mathrm{H}$ NMR spectrum of $\mathbf{O}_{4}-\mathbf{m}-\mathbf{O}_{4}\left(500 \mathrm{MHz}, \mathrm{CDCl}_{3}\right)$.
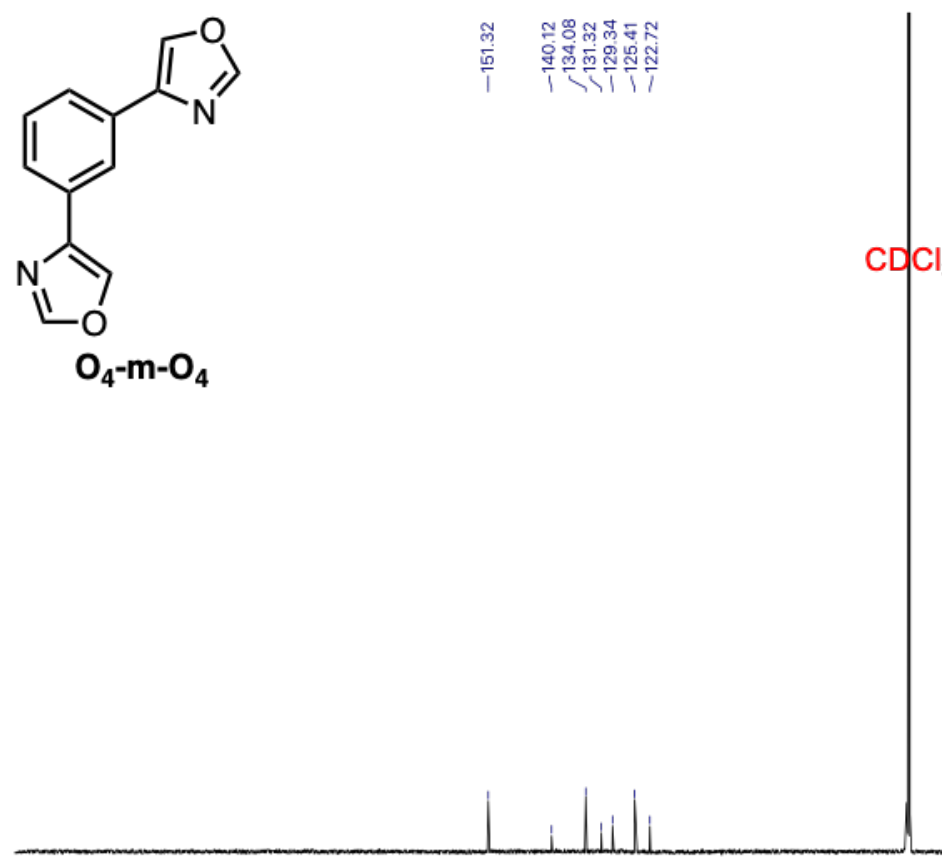

200

150

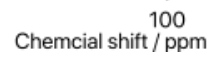

50

0

Figure S8. ${ }^{13} \mathrm{C}$ NMR spectrum of $\mathbf{O}_{4}-\mathbf{m}-\mathbf{O}_{4}\left(125 \mathrm{MHz}, \mathrm{CDCl}_{3}\right)$. 
<smiles>N#Cc1ccc(-c2cocn2)cc1</smiles>

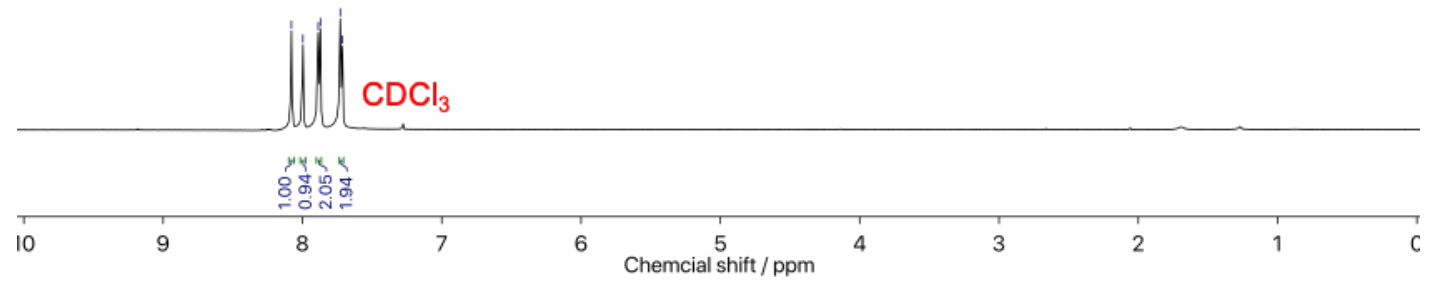

Figure S9. ${ }^{1} \mathrm{H} \mathrm{NMR}$ spectrum of $\mathbf{1}\left(500 \mathrm{MHz}, \mathrm{CDCl}_{3}\right)$.<smiles>N#Cc1ccc(-c2cocn2)cc1</smiles>

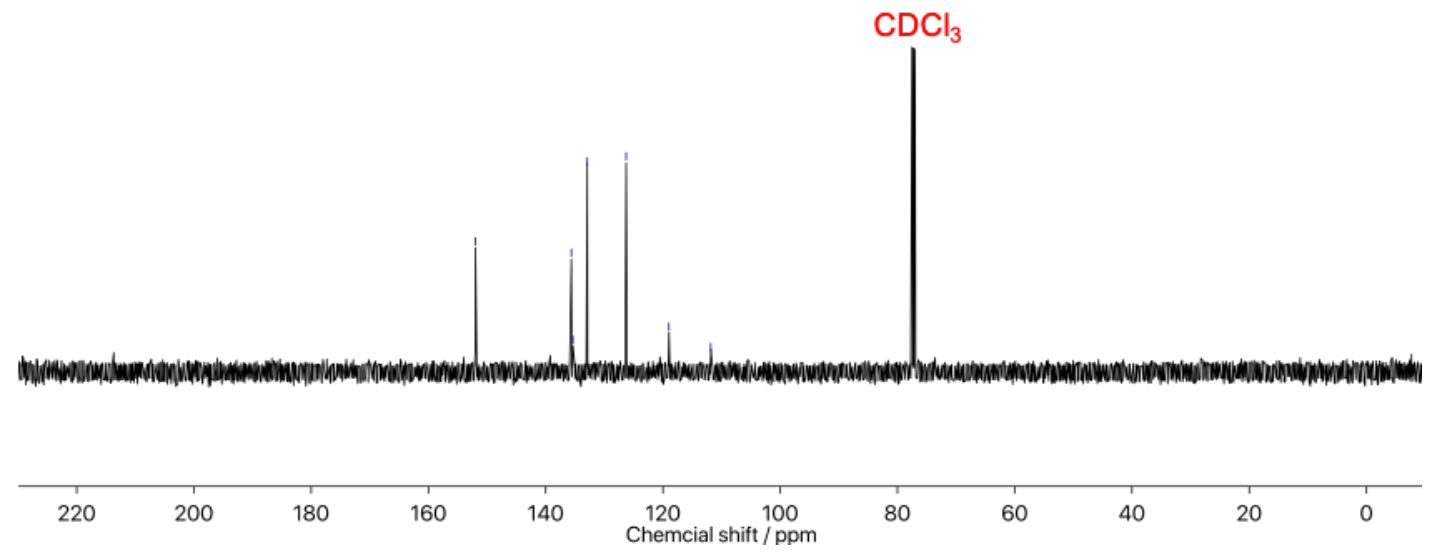

Figure S10. ${ }^{13} \mathrm{C}$ NMR spectrum of $1\left(125 \mathrm{MHz}, \mathrm{CDCl}_{3}\right)$. 

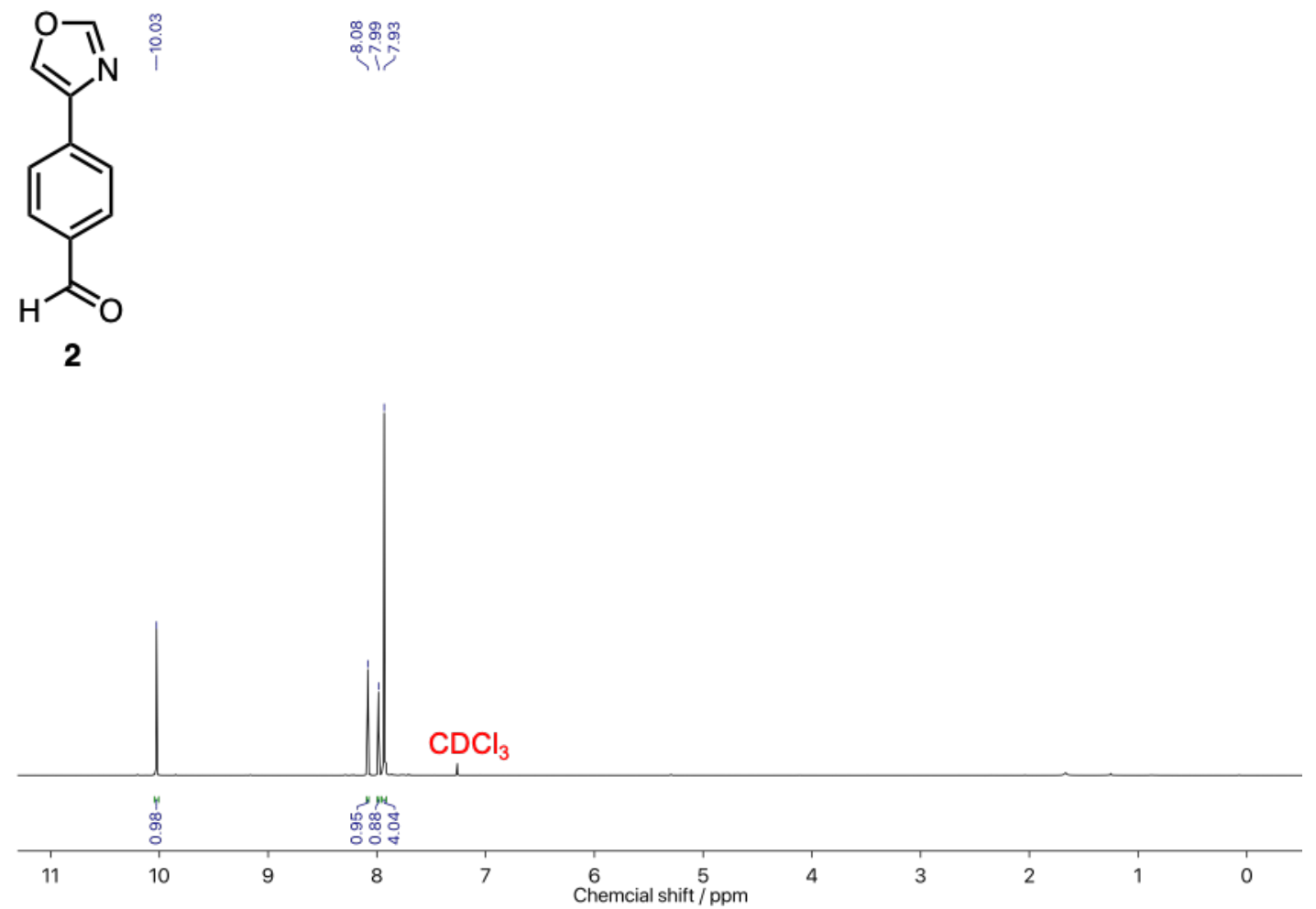

Figure S11. ${ }^{1} \mathrm{H} \mathrm{NMR}$ spectrum of $2\left(500 \mathrm{MHz}, \mathrm{CDCl}_{3}\right)$.
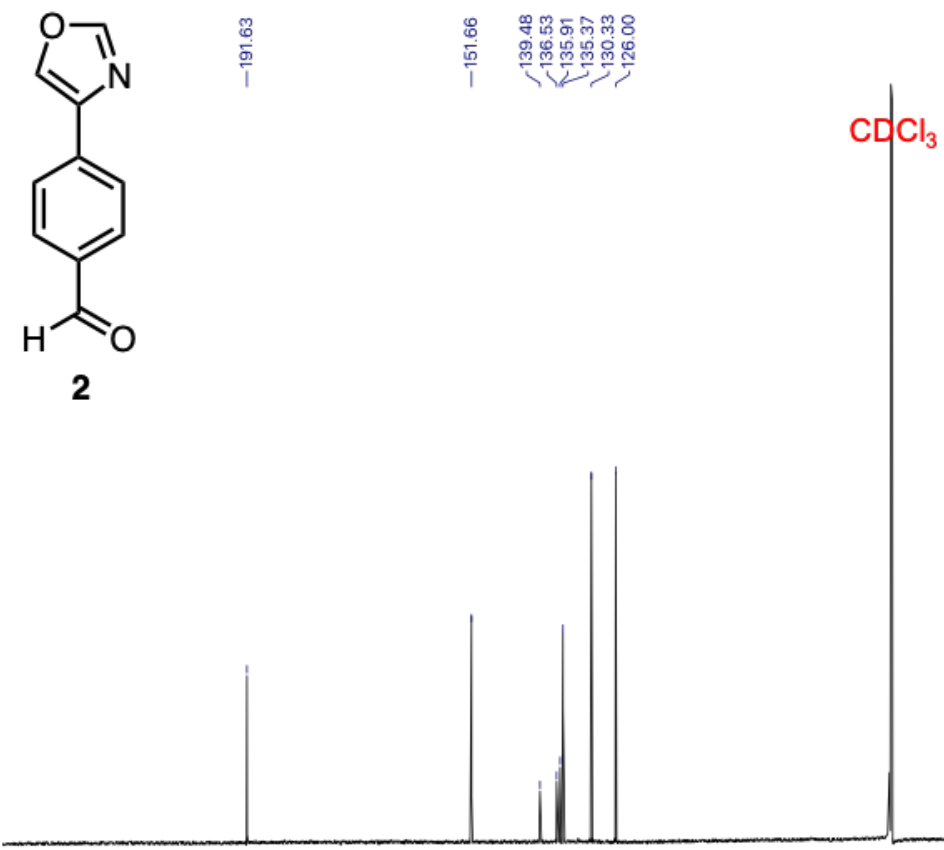

200
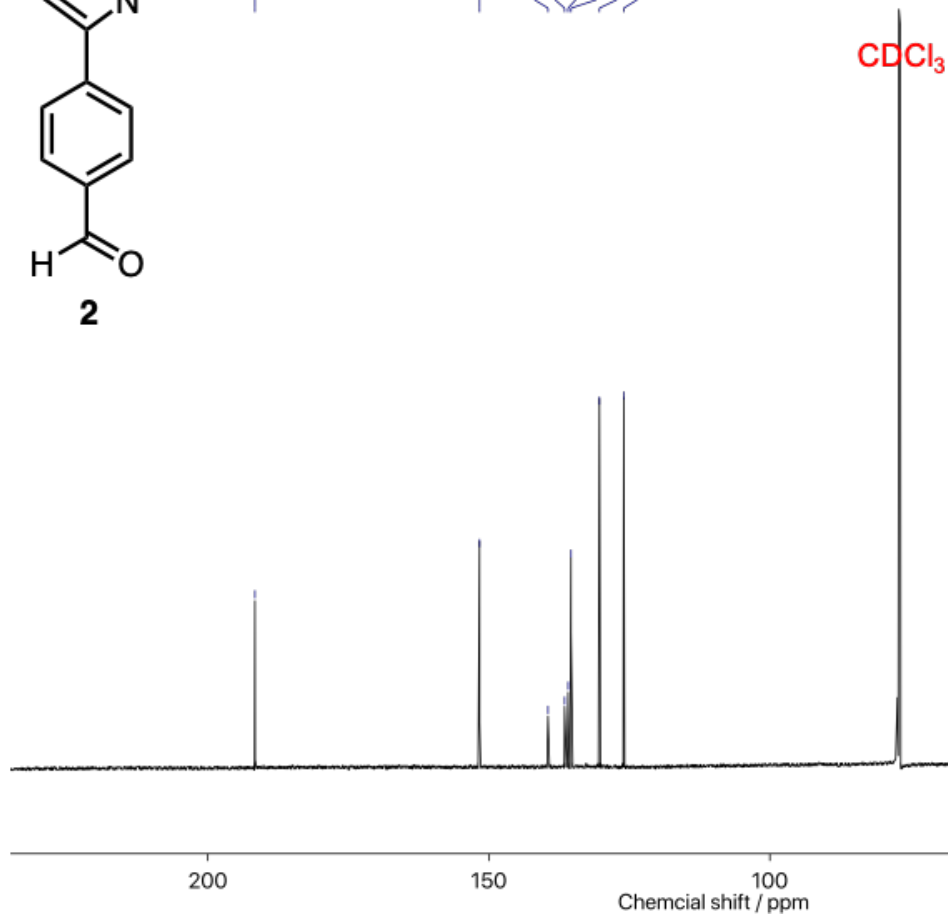

150

Chemcial shift / ppm

50

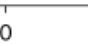

Figure S12. ${ }^{13} \mathrm{C}$ NMR spectrum of $2\left(125 \mathrm{MHz}, \mathrm{CDCl}_{3}\right)$. 

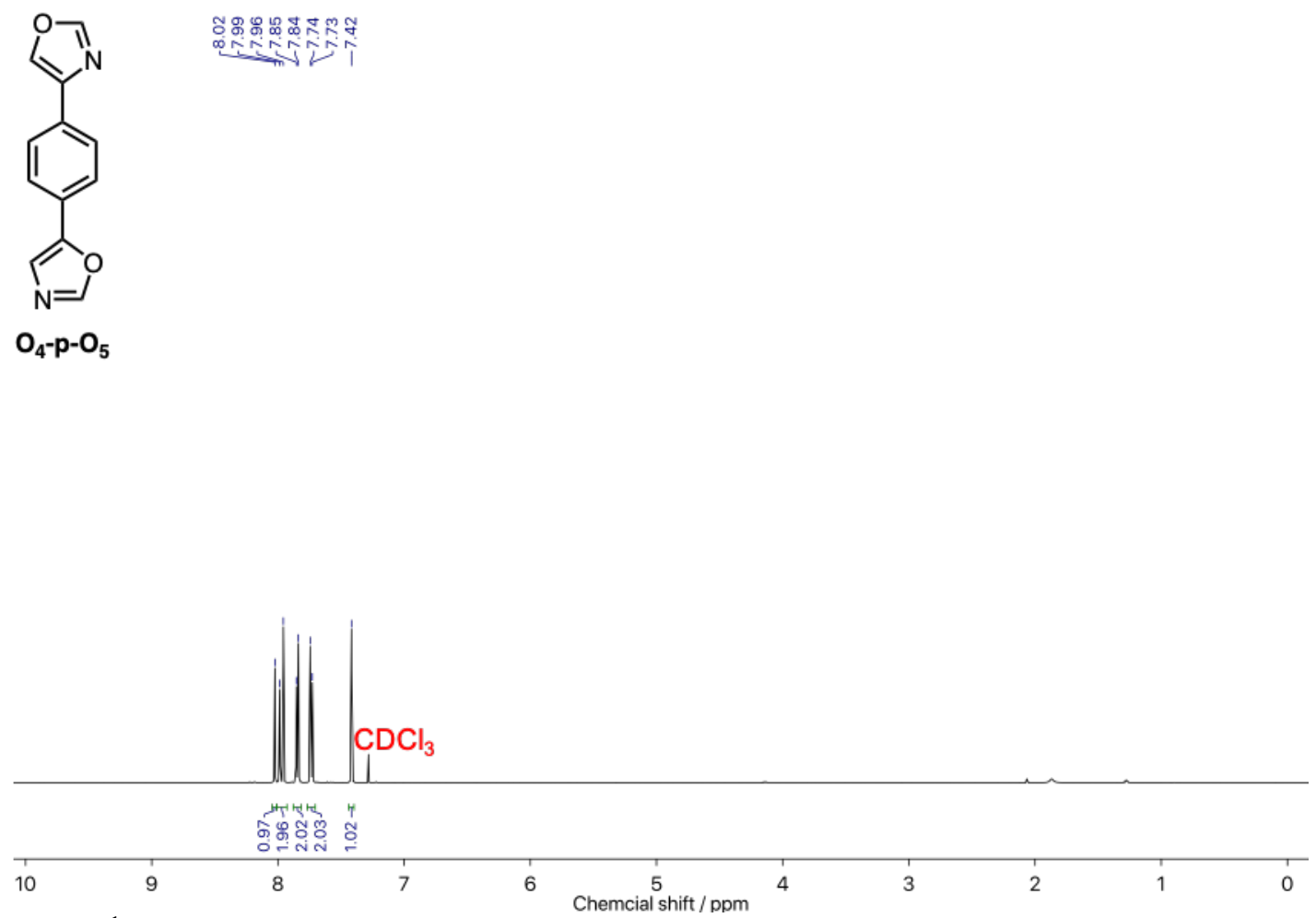

Figure S13. ${ }^{1} \mathrm{H}$ NMR spectrum of $\mathbf{O}_{4}-\mathbf{p}-\mathbf{O}_{\mathbf{5}}\left(500 \mathrm{MHz}, \mathrm{CDCl}_{3}\right)$.
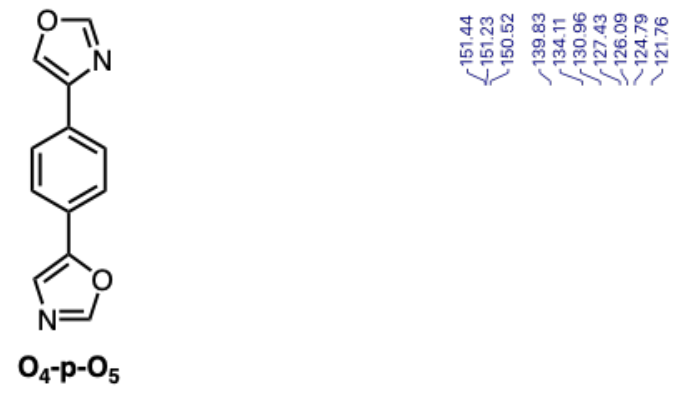

$\mathrm{O}_{4-p-\mathrm{O}_{5}}$
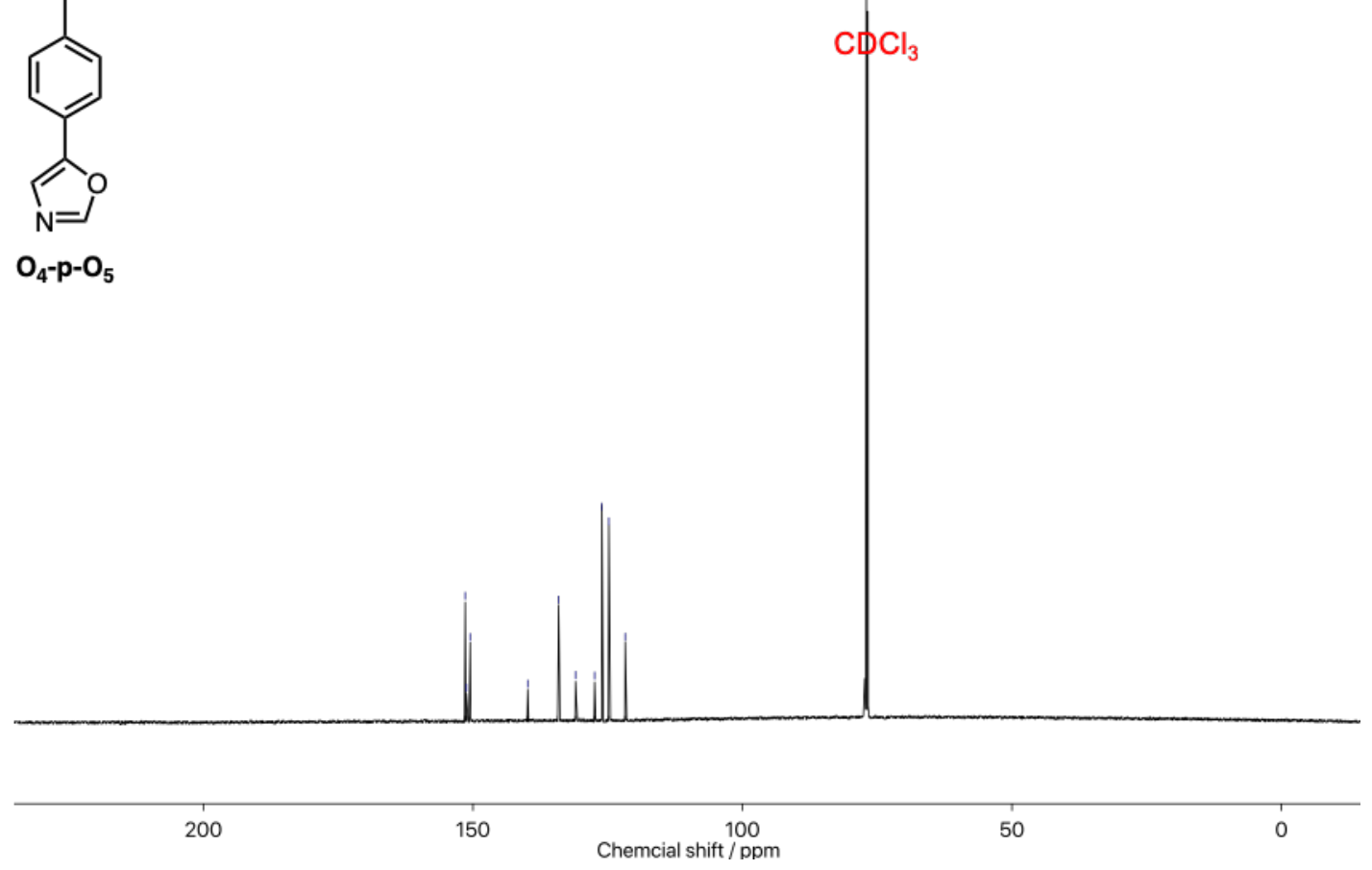

Figure S14. ${ }^{13} \mathrm{C}$ NMR spectrum of $\mathbf{O}_{4}-\mathbf{p}-\mathbf{O}_{5}\left(125 \mathrm{MHz}, \mathrm{CDCl}_{3}\right)$. 


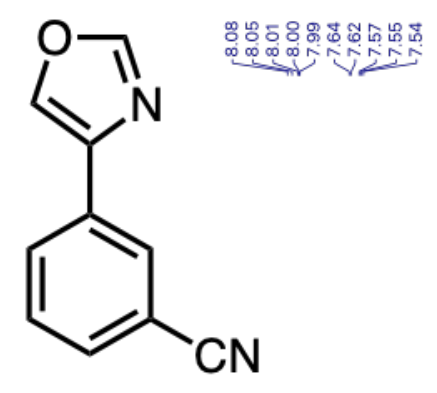

3

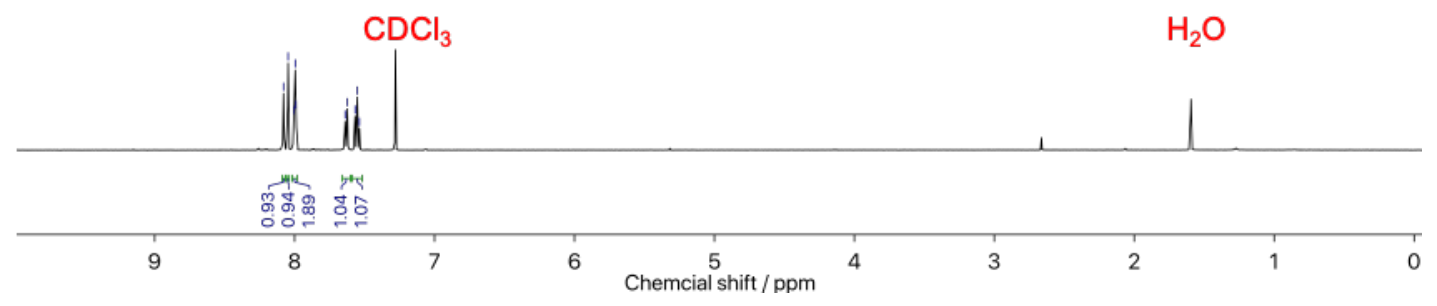

Figure S15. ${ }^{1} \mathrm{H}$ NMR spectrum of $3\left(500 \mathrm{MHz}, \mathrm{CDCl}_{3}\right)$.

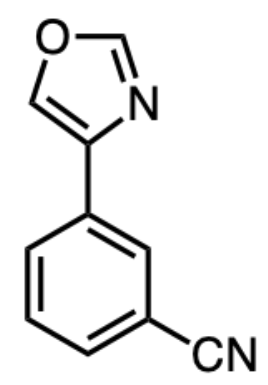

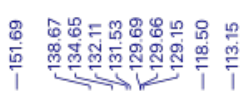

3

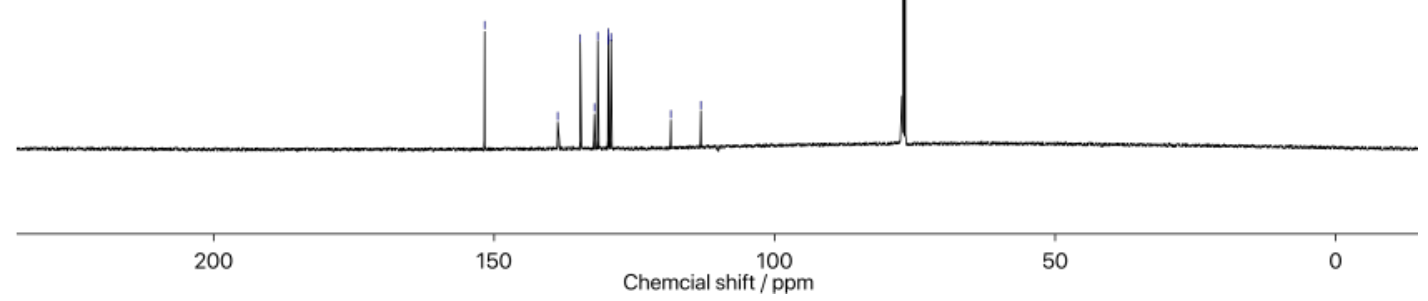

Figure S16. ${ }^{13} \mathrm{C}$ NMR spectrum of $3\left(125 \mathrm{MHz}, \mathrm{CDCl}_{3}\right)$. 


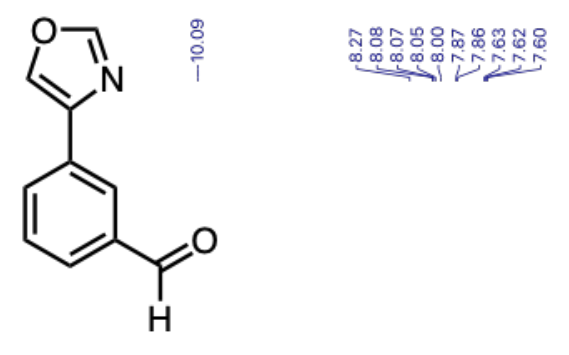

4

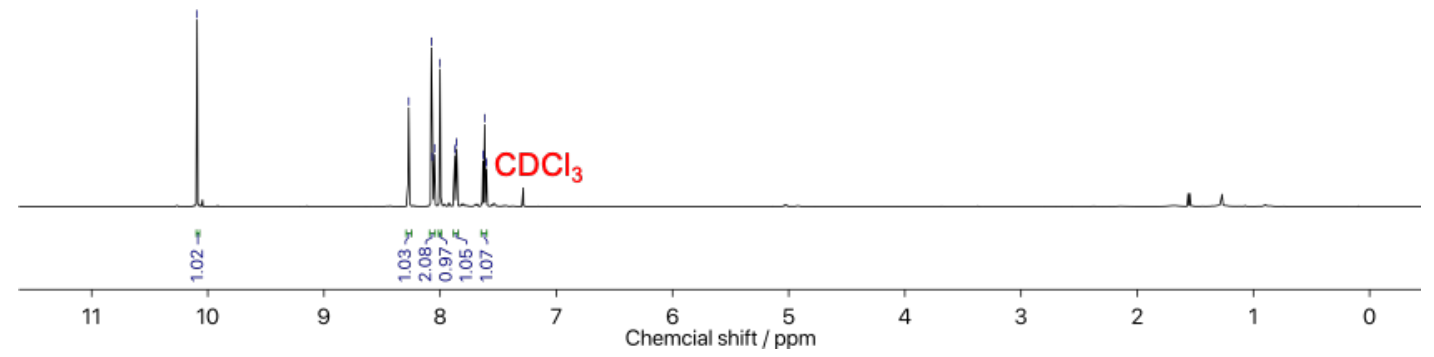

Figure S17. ${ }^{1} \mathrm{H}$ NMR spectrum of $4\left(500 \mathrm{MHz}, \mathrm{CDCl}_{3}\right)$.

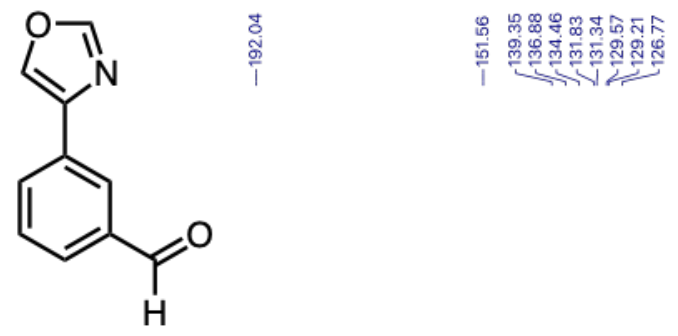

4

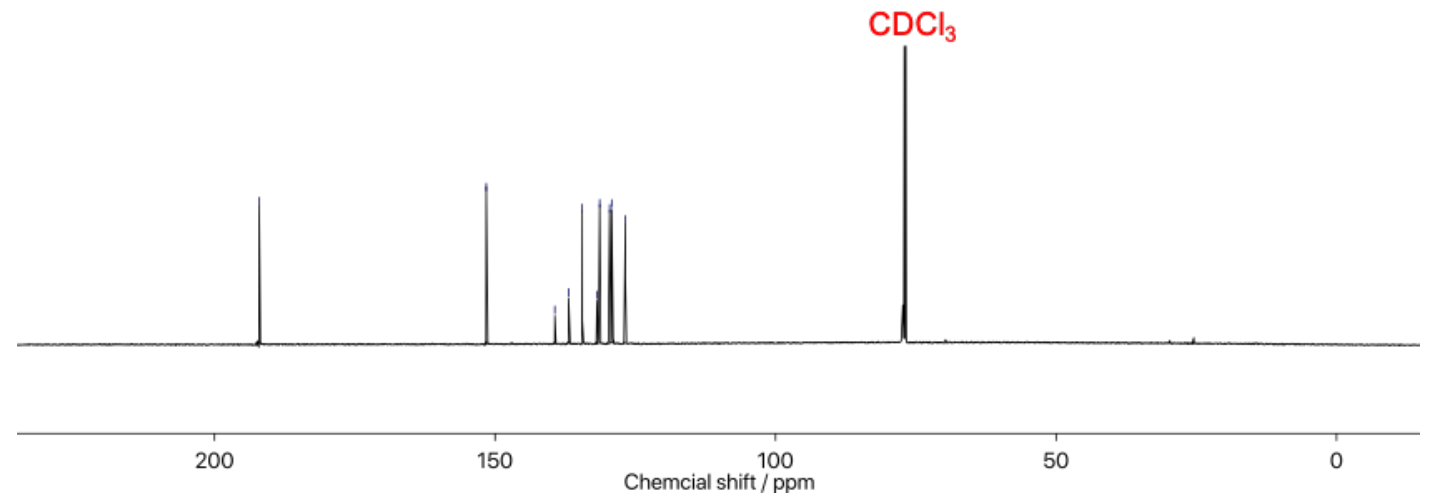

Figure S18. ${ }^{13} \mathrm{C}$ NMR spectrum of $4\left(125 \mathrm{MHz}, \mathrm{CDCl}_{3}\right)$. 


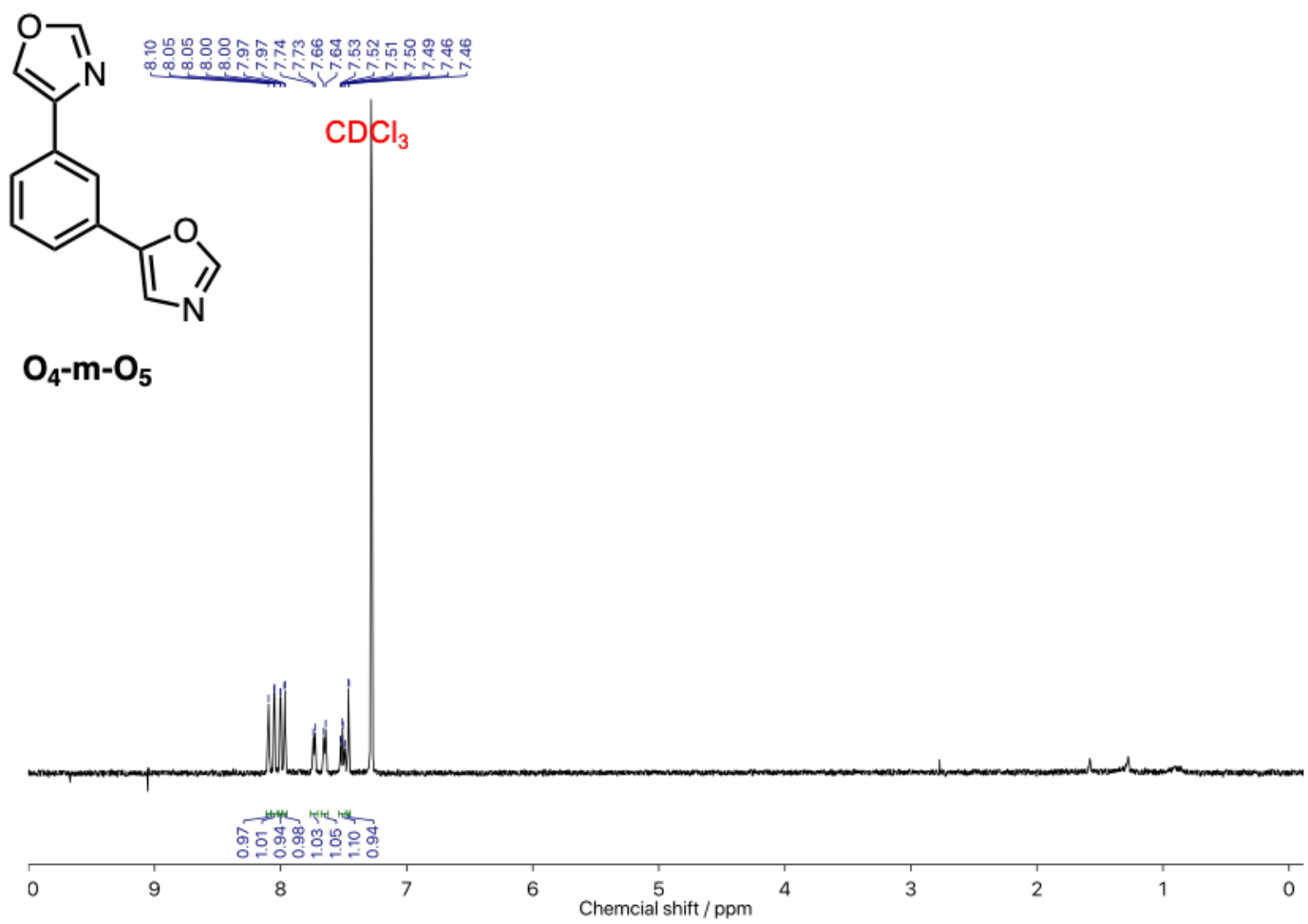

Figure S19. ${ }^{1} \mathrm{H}$ NMR spectrum of $\mathbf{O}_{4}-\mathbf{m}-\mathbf{O}_{5}\left(500 \mathrm{MHz}, \mathrm{CDCl}_{3}\right)$.<smiles>c1cc(-c2cnco2)cc(-c2cnco2)c1</smiles>

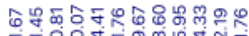

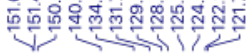
$\mathrm{O}_{4}-\mathrm{m}-\mathrm{O}_{5}$

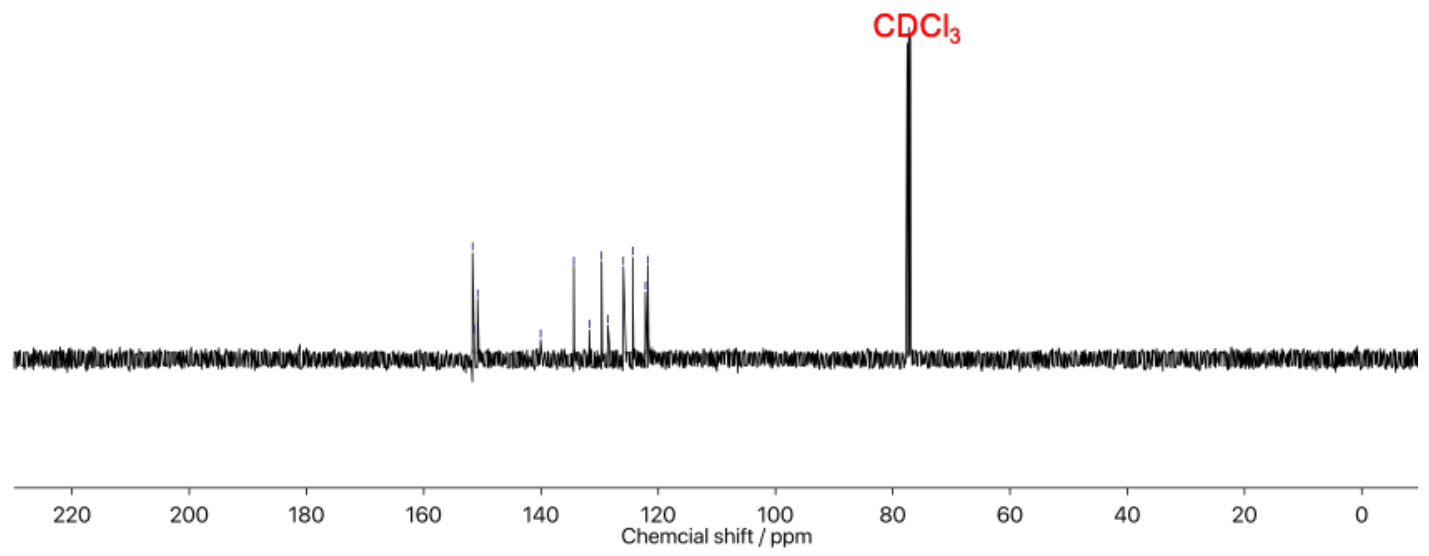

Figure S20. ${ }^{13} \mathrm{C}$ NMR spectrum of $\mathbf{O}_{4}-\mathbf{m}-\mathbf{O}_{5}\left(125 \mathrm{MHz}, \mathrm{CDCl}_{3}\right)$. 


\section{S.4 ${ }^{1} \mathrm{H}$ and ${ }^{13} \mathrm{C}$ NMR Predications of OX2}

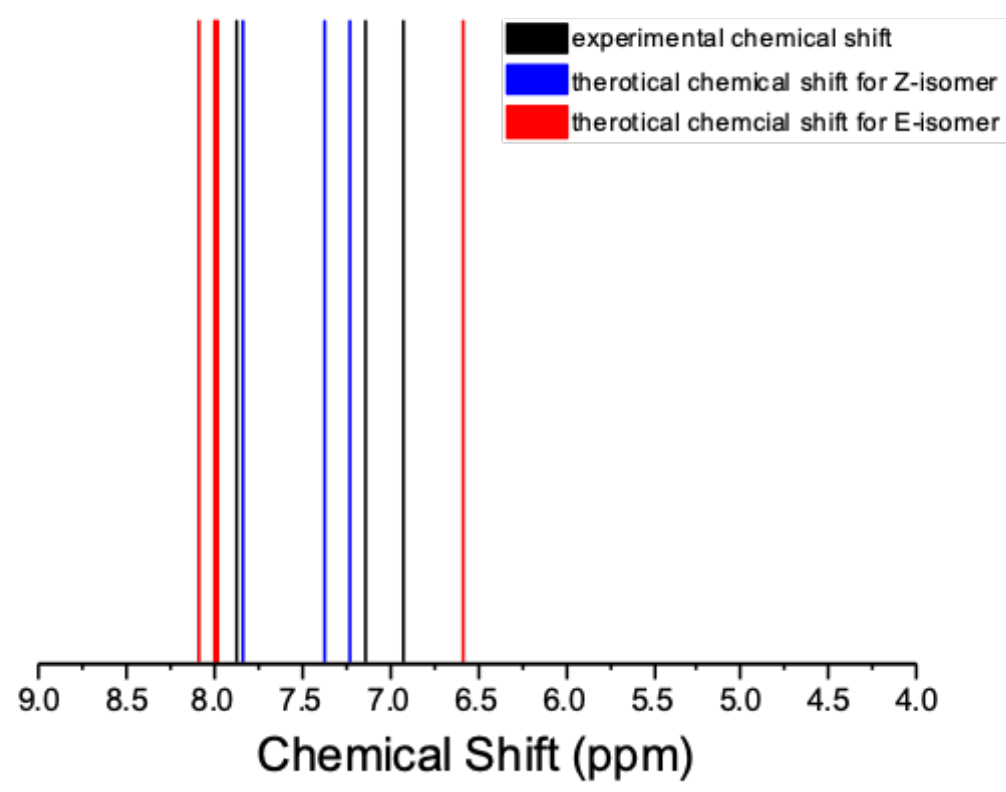

Figure S21. Comparison of ${ }^{1} \mathrm{H}$ NMR spectra and theoretical NMR pattern (OX2). Attempts to obtain a crystal structure of $\mathbf{O X} \mathbf{2}$ were not successful. The ${ }^{1} \mathrm{H}$ NMR pattern of the $E$-isomer appears to be more consistent with the experimental data (black bars) compared to the Z-isomer. Therefore, we conjecture that $\mathbf{O X 2}$ appeared as the trans-isomer. The ${ }^{1} \mathrm{H}$ NMR chemical shifts were calculated using Spartan '16. The geometries are optimized at the B3LYP/6-311G(d) level of theory, and the ${ }^{1} \mathrm{H}$ NMR chemical shifts are calculated at the same level. The computed chemical shift was calibrated using $\mathrm{SiMe}_{4}(0.00 \mathrm{ppm}), \mathrm{CH}_{2} \mathrm{Cl}_{2}(5.35 \mathrm{ppm})$, and $\mathrm{CHCl}_{3}(7.26 \mathrm{ppm})$, which yielded a linear calibration curve: $\delta_{\exp }=1.0548 \delta_{\text {calc }}-0.0017\left(R^{2}=1\right)$. This calibration curve was then applied to the calculated shifts of protons in the $Z$ - (red bars) and $E$-isomer (blue bars) of OX2. 


\section{S.5 Additional Experimental Data and Theoretical Calculations}

Conductance Measurements

All single molecule conductance measurements were performed in a home-built scanning tunneling microscope setup, using a method as previously described. ${ }^{55-7}$ Gold STM insulated tips were prepared using $0.25 \mathrm{~mm}$ Au wire $(99.998 \%$, Alfa Aesar). Gold substrates were prepared by evaporating $120 \mathrm{~nm}$ of gold onto polished AFM metal specimen discs (Ted Pella). Conductance measurements were carried out in $1 \mathrm{mM}$ molecule solution in 1,2,4-trichlorobenzene. The break junction experiments were conducted at a bias of $250 \mathrm{mv}$. All histograms (over 10,000 traces for each molecule) are constructed without any data selection.

\section{Theoretical Calculations}

Electron transport calculations were calculated using nonequilibrium Green's function/density functional theory method via the Atomistix Toolkit package. ${ }^{\mathrm{S} 8-10} \mathrm{We}$ optimize all molecules using Spartan with a 6-31 G** basis. Then we place the optimized molecules in built junctions and relax all atoms to $0.05 \mathrm{ev} / \AA$ using DFT with local spin density approximation, a double- $\zeta$ polarized basis set for the molecules except for gold atoms with a single- $\zeta$ basis set, and k-point samplings of $3 \mathrm{x}$ $3 \times 50$ (with 50 being in the direction along the transport). Finally, we calculated the transmission spectra through the junction. 

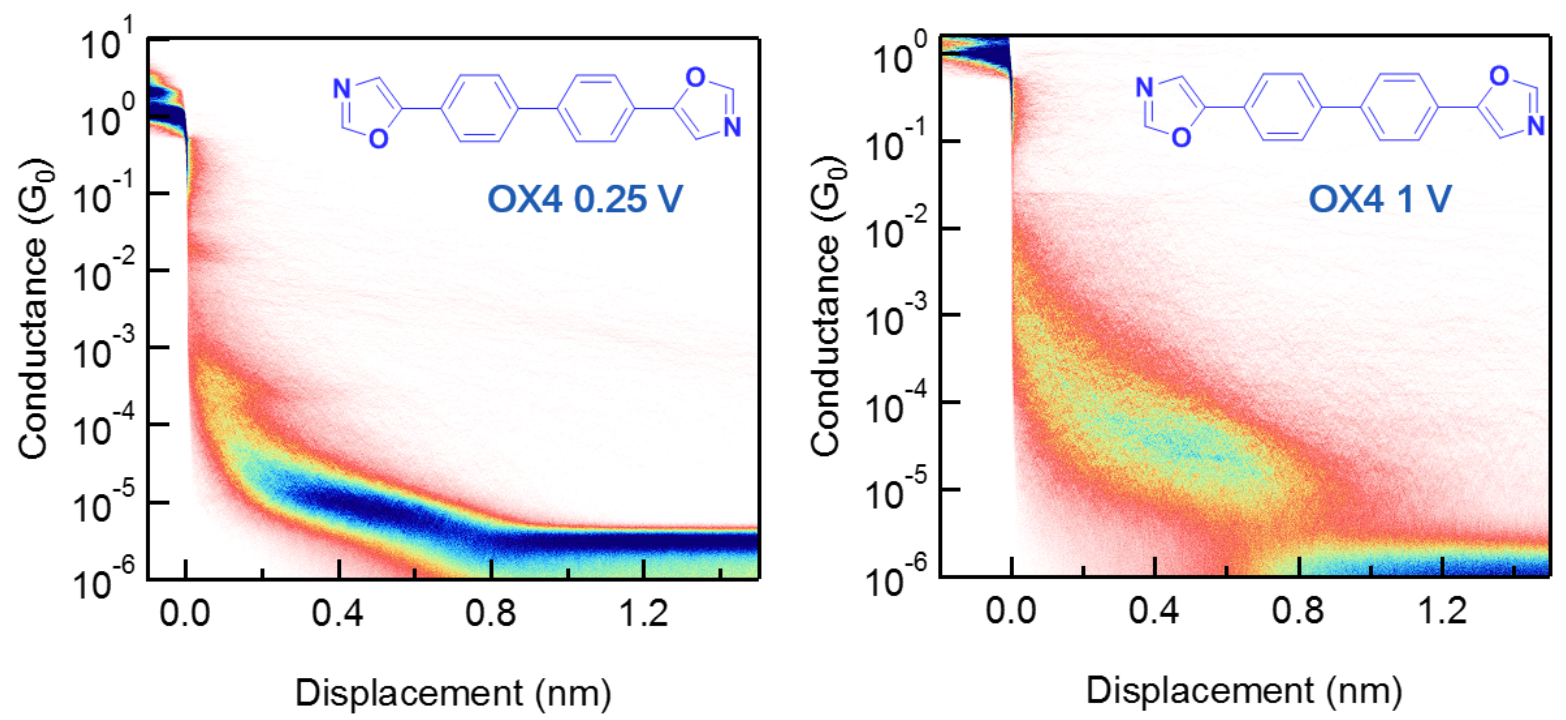

Figure S22. 2D conductance histograms for OX4 at $0.25 \mathrm{~V}$ and $1 \mathrm{~V}$
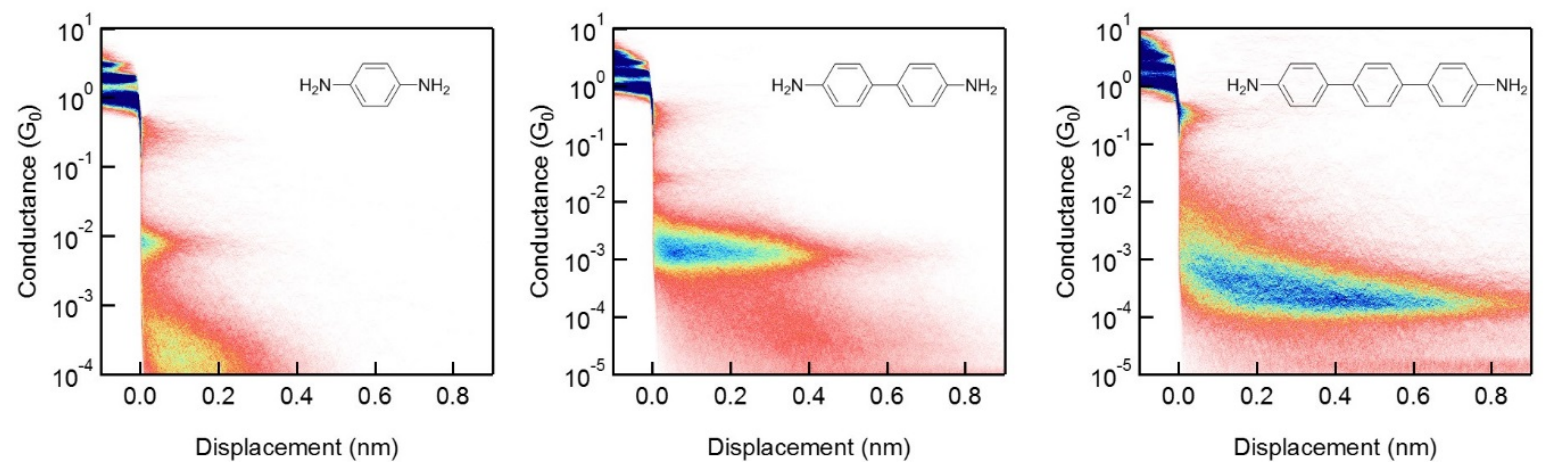

Figure S23. 2D conductance histograms for oligophenyls at $0.25 \mathrm{~V}$

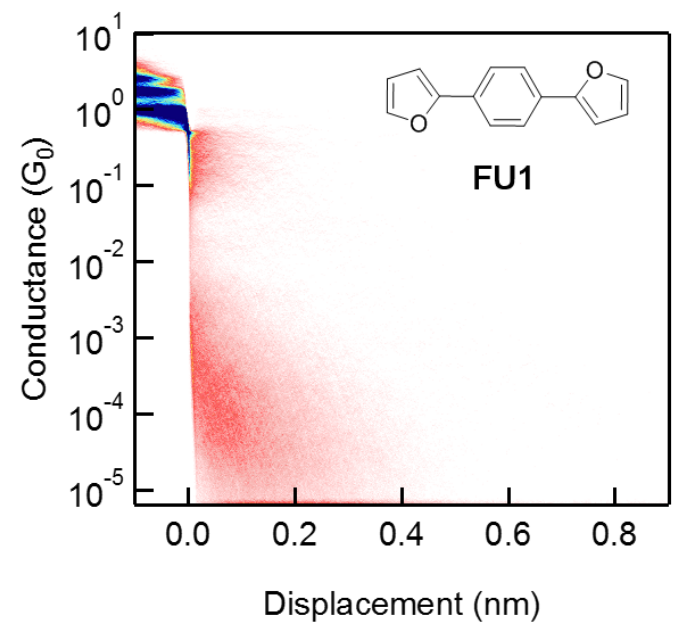

Figure S24. 2D conductance histograms for FU1 at $0.25 \mathrm{~V}$ 

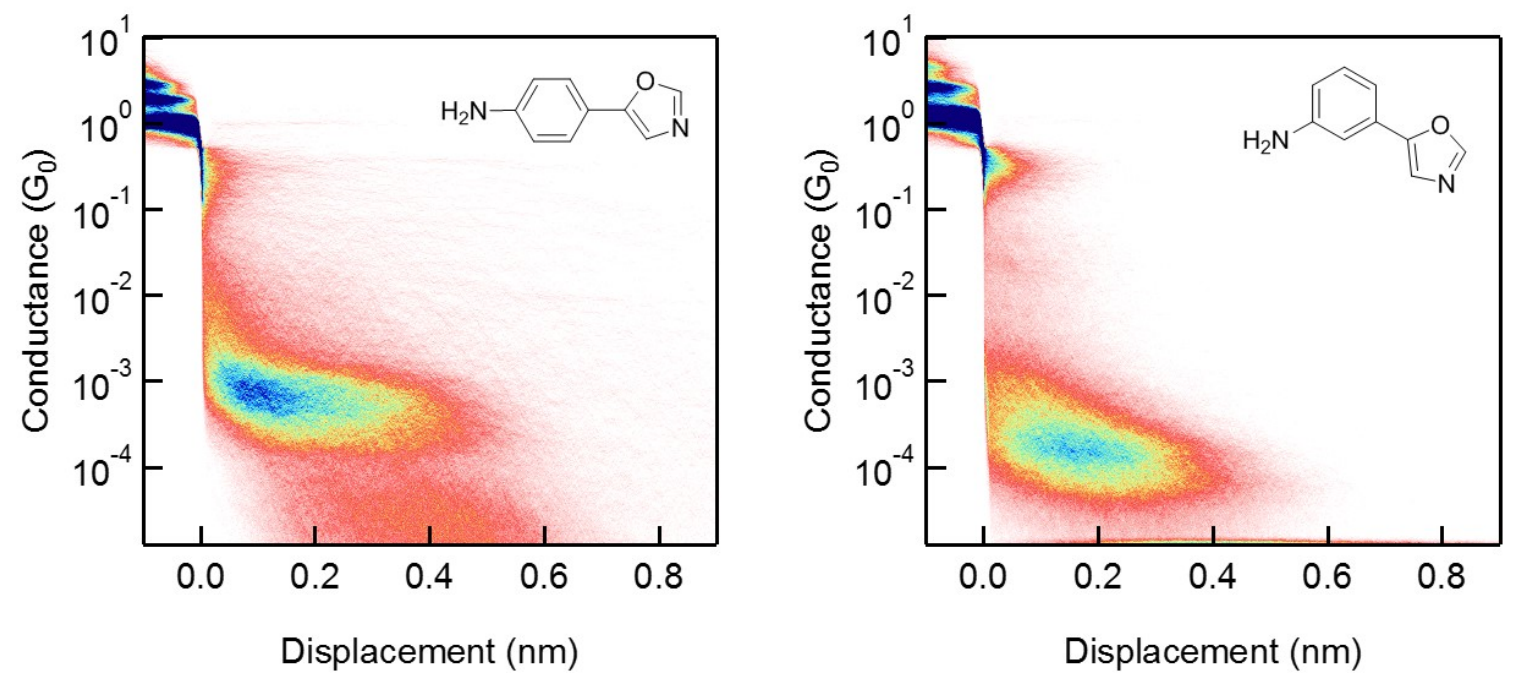

Figure S25. 2D conductance histograms for A-p- $\mathrm{O}_{5}$ and A-m- $\mathrm{O}_{5}$ at $0.25 \mathrm{~V}$
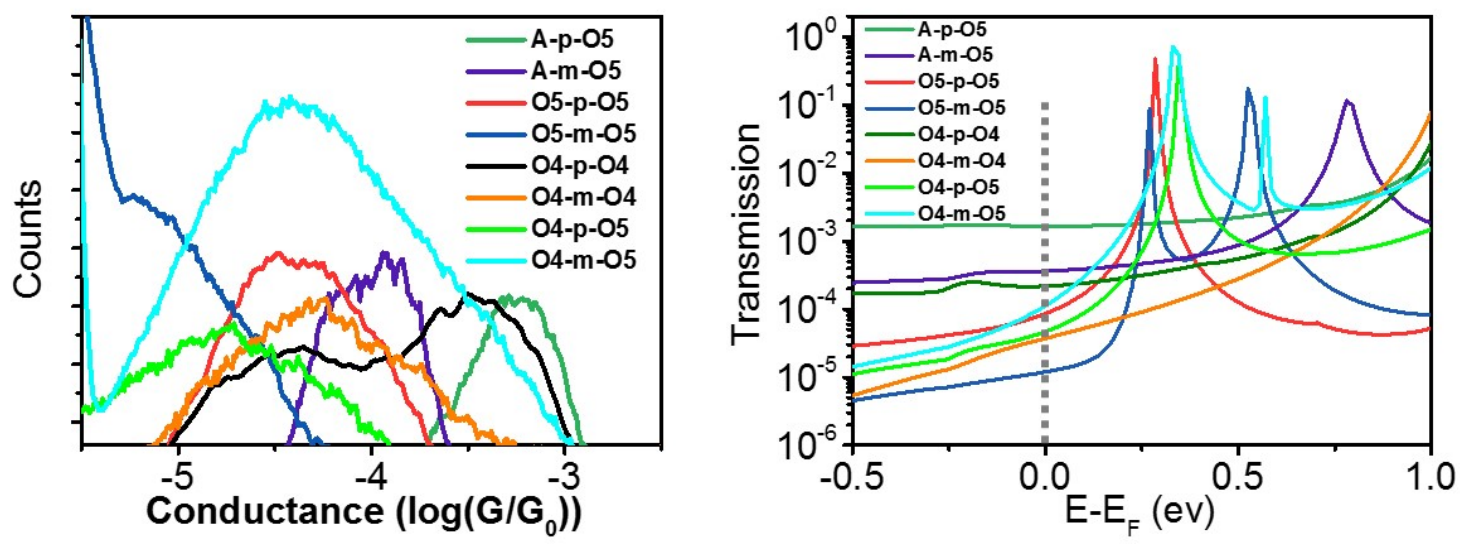

Figure S26. Conductance histograms ( $0.25 \mathrm{~V}$ bias voltage for each histogram) and transmission spectrum for oxazole-terminated derivatives. 

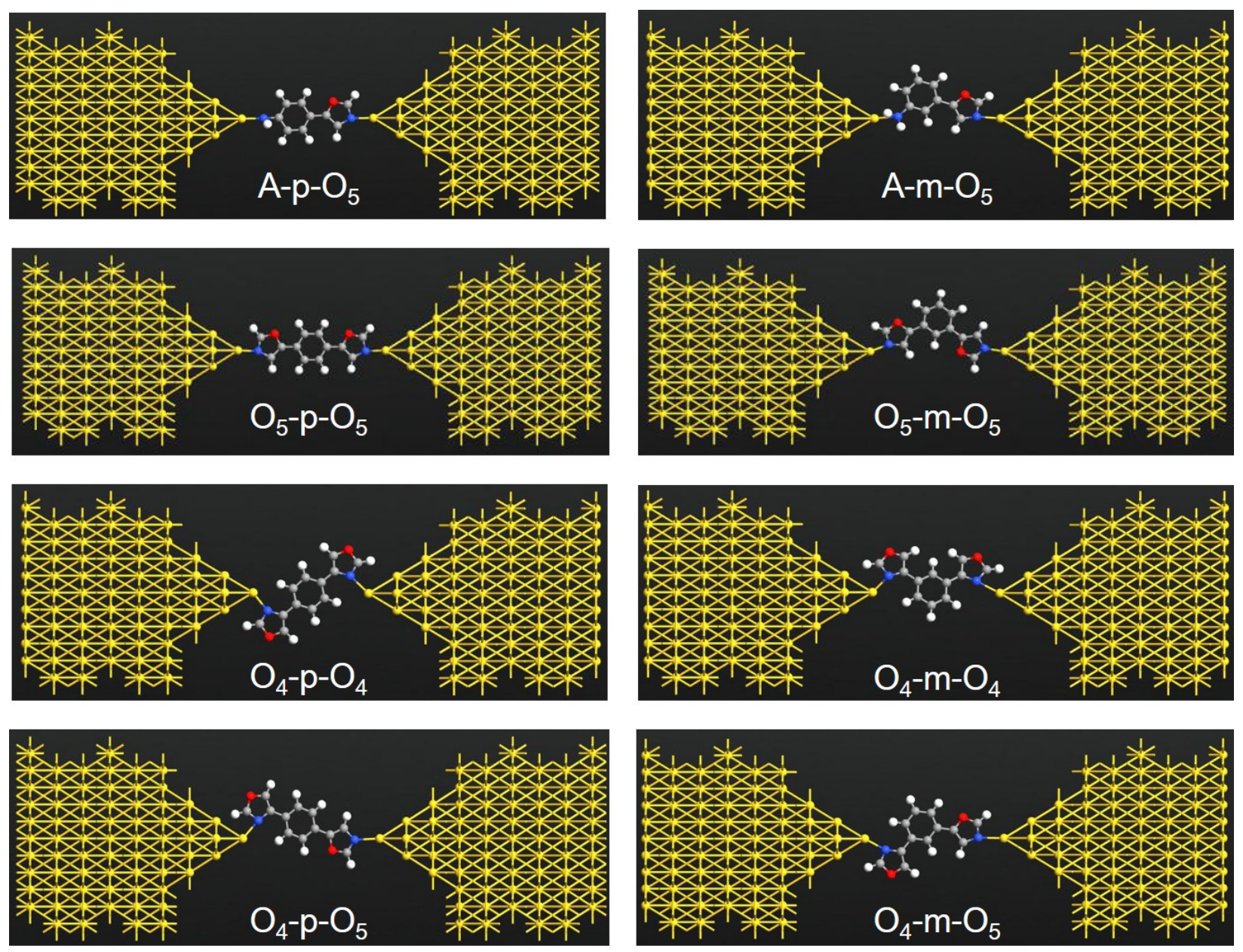

Figure S27. Structures of molecular junctions in NEGF-DFT simulations for oxazole-terminated derivatives.

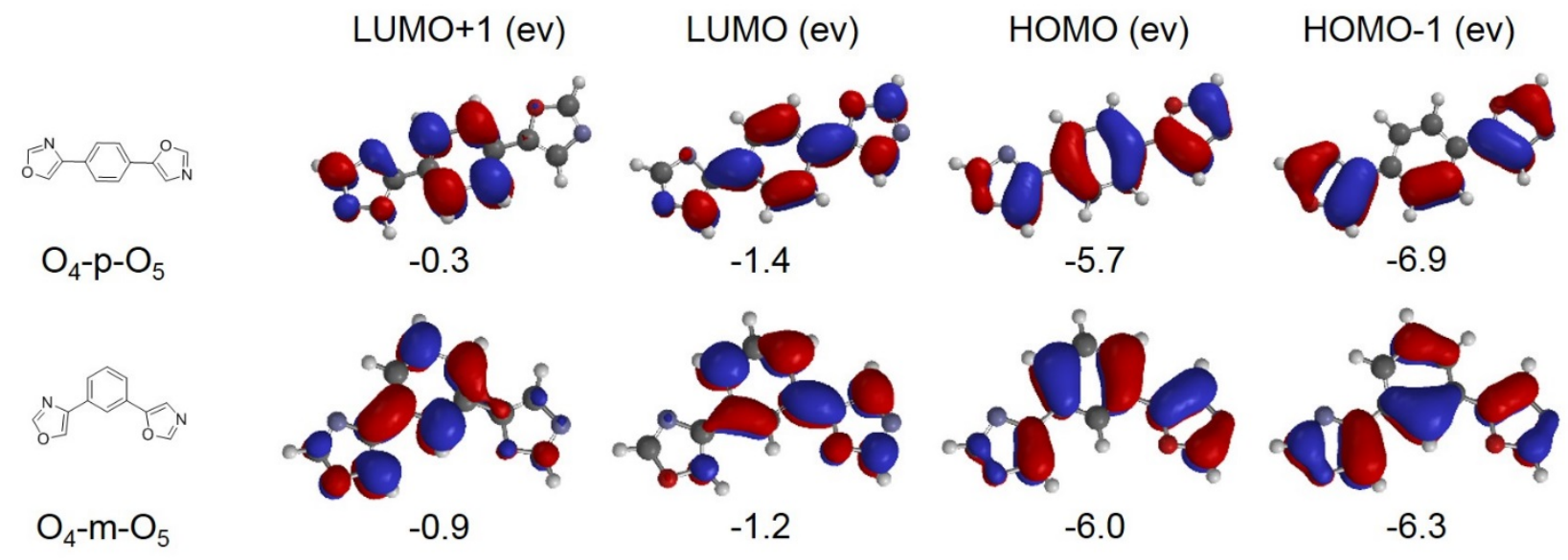

Figure S28. Molecular orbitals (isosurfaces) and calculated energies for $\mathrm{O}_{4}-\mathrm{p}-\mathrm{O}_{5}$ and $\mathrm{O}_{4}-\mathrm{m}-\mathrm{O}_{5}$ 


\section{References}

(1) Kotha, S.; Shah, V. R. Synthesis of Bis- and Trisoxazole Derivatives via SuzukiMiyaura Cross-Coupling Reaction and van Leusen Oxazole Synthesis. Synthesis 2007, 2007, 3653-3658.

(2) Nadres, E. T.; Lazareva, A.; Daugulis, O. Palladium-Catalyzed Indole, Pyrrole, and Furan Arylation by Aryl Chlorides. J. Org. Chem. 2011, 76 (2), 471-483.

(3) Primas, N.; Bouillon, A.; Lancelot, J.-C.; Rault, S. Synthesis of 2-TIPS-Oxazol-5Ylboronic Acid Pinacol Ester: Efficient Route to 5-(Het)aryloxazoles via Suzuki CrossCoupling Reaction. Tetrahedron 2009, 65 (32), 6348-6353.

(4) Proskelia SAS; WO 20070600262007

(5) Venkataraman, L.; Klare, J. E.; Nuckolls, C.; Hybertsen, M. S.; Steigerwald, M. L. Nature 2006, 442, 904.

(6) Garner, M. H.; Li, H.; Chen, Y.; Su, T. A.; Shangguan, Z.; Paley, D. W.; Liu, T.; Ng, F.; Li, H.; Xiao, S. Nature 2018, 1.

(7) Zang, Y.; Ray, S.; Fung, E.-D.; Borges, A.; Garner, M. H.; Steigerwald, M. L.; Solomon, G. C.; Patil, S.; Venkataraman, L. Journal of the American Chemical Society 2018, 140, 13167.

(8) Cai, Z.; Lo, W.-Y.; Zheng, T.; Li, L.; Zhang, N.; Hu, Y.; Yu, L. Journal of the American Chemical Society 2016, 138, 10630.

(9) Atomistix ToolKit version 2017.12 (QuantumWise A/S, 2017); www.quantum wise.com.

(10) Virtual NanoLab version 2017.12 (QuantumWise A/S, 2017); www.quantumwise.com. 